Aus dem Universitätsklinikum Münster

Klinik und Poliklinik für Anästhesiologie und operative Intensivmedizin

- Direktor: Univ.-Prof. Dr. med. Dr. h. c. Hugo Van Aken und dem Institut für Hygiene

- Direktor: Univ.-Prof. Dr. rer. nat. Helge Karch

\title{
Rückschlagventile und bakterielle Kontamination
}

\author{
INAUGURAL-DISSERTATION \\ zur \\ Erlangung des doctor medicinae \\ der Medizinischen Fakultät der \\ Westfälischen Wilhelms-Universität Münster \\ vorgelegt von \\ Kiski, Daniela \\ aus Haltern am See \\ 2009
}


Gedruckt mit freundlicher Genehmigung der Medizinischen Fakultät der Westfälischen Wilhelms-Universität Münster 
Dekan: Univ.-Prof. Dr. med. Wilhelm Schmitz

1.Berichterstatter: Priv.-Doz. Dr. med. B. Ellger

2.Berichterstatter: Priv.-Doz. Dr. med. A. Friedrich

Tag der mündlichen Prüfung: 14.09.2009 
Aus dem Universitätsklinikum Münster

Klinik und Poliklinik für Anästhesiologie und operative Intensivmedizin

- Direktor: Univ.-Prof. Dr. med. Dr. h.c. Hugo Van Aken, FRCA, FANZCA und dem Institut für Hygiene

- Direktor: Univ.-Prof. Dr. rer. nat. Helge Karch

Referent: Priv.-Doz. Dr. med. B. Ellger

Co-Referent: Priv.-Doz. Dr. med. A. Friedrich

\section{Zusammenfassung}

\section{Rückschlagventile und bakterielle Kontamination}

Kiski, Daniela

Rückschlagventile (RVs) sollen eine Volumenverschiebung gegen die Durchflussrichtung verhindern, um bei Schwerkraft- und Druckinfusionen eine Vermischung parallel verabreichter Infusionslösungen zu verhindern. Wir untersuchten, ob RVs bei Langzeitinfusionen einen Rückfluss von Infusionslösungen und damit eine bakterielle Kontamination vermeiden können.

In unserem Experiment wurde im ersten Teil das Schließverhalten von 40 RVs 5 verschiedener RV-Modelle analysiert. Bei pumpengetriebener Infusion gegen die RV-Durchflussrichtung solte sich, bei Schluss des RVs, durch Volumenverschiebung vor dem RV ein Druck linear erhöhen. Im zweiten Teil wurde untersucht, ob RVs eine bakterielle Kontamination einer Druckleitung verhindern. Hierbei wurden Indikatorbakterien mit unterschiedlichem Migrationsverhalten (S. aureus, S. epidermidis, P. mirabilis) in unterschiedlichen Infusionslösungen ( $\mathrm{NaCl} 0.9 \%$ und Propofol) untersucht. Getestet wurde das Migrationsverhalten der Bakterien bei Druckaufbau gegen die RVDurchflussrichtung sowie in Durchflussrichtung.

Bei einer Perfusorförderrate von $0,1 \mathrm{ml} / \mathrm{h}$ und Druckaufbau gegen die RVDurchflussrichtung waren 14\% der Ventile undicht. Bei einer Förderrate von 1 $\mathrm{ml} / \mathrm{h} 7,5 \%$. Alle untersuchten Bakterienstämme konnten in der Infusionslösung Propofol die RVs passieren. In $\mathrm{NaCl} 0.9 \%$ passierte nur $P$. mirabilis das RV.

Die Arbeit zeigt, dass RVs einen Rückfluss von Infusionslösungen nicht zuverlässig verhindern und keinen sicheren Schutz gegen bakterielle Kontamination bieten. 


\section{Inhaltsverzeichnis}

1. Einleitung und Problemstellung..........................................

1.1. Funktion von Rückschlagventilen (RVs) in der Medizin....................1

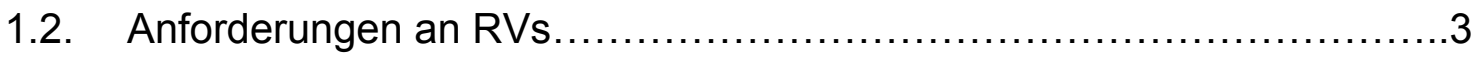

1.2.1. Verhinderung von Rückfluss in das Infusionssystem ...............3

1.2.2. Allgemeine Qualitätsanforderungen an RVs.........................

1.2.3. Schutz vor Kontamination durch RVs.............................. 4

1.3. Studienziel und Hypothese ............................................. 5

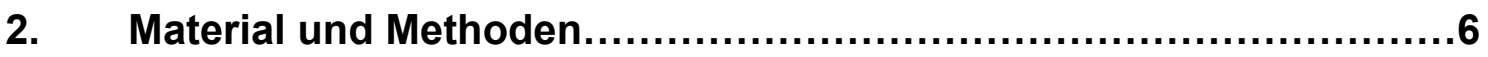

2.1. Material und Methoden der physikalischen Testung.......................6

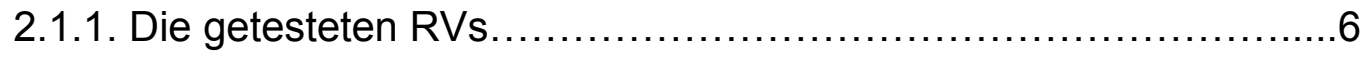

2.2. Testung des Schließverhaltens der RVs ...............................

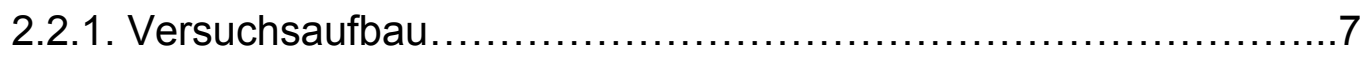

2.2.2. Versuchsablauf.................................................. 8

2.3. Material und Methoden der mikrobiologischen Testung................... 9

2.3.1. Die getesteten Bakterienstämme................................. 9

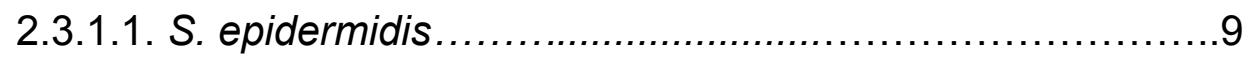

2.3.1.2. S. aureus.................................................... 10

2.3.1.3. P. mirabilis .................................................... 10

2.3.2. Die getesteten Infusionslösungen ............................... 11

2.3.2.1. Propofol.................................................. 11

2.3.2.2. $\mathrm{NaCl} 0.9 \%$-Lösung ........................................11

2.3.3. Testung der Verhinderung bakterieller Kontamination durch

RVs bei Infusion gegen die RV-Durchflussrichtung................12

2.3.3.1. Versuchsablauf.............................................

2.3.4. Testung bakterieller Kontamination retrograd über RVs gegen einen Flüssigkeitsstrom in RV-Durchflussrichtung...........16

2.3.4.1. Versuchsablauf...............................................16 


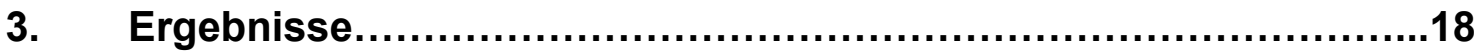

3.1. Ergebnisse der physikalischen Testung der RVs..................... 18

3.1.1. Referenzmessungen......................................

3.1.2. Analyse der Messergebnisse................................ 19

3.1.2.1. Analyse der Steigungsbeginne (Min.) der

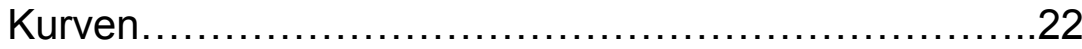

3.1.2.2. Analyse der Maximaldrücke $(\mathrm{mmHg})$ am Versuchsende

3.1.2.3. Analyse der Lage der Kurven im Vergleich zur Referenzgeraden..................................24

3.1.2.4. Analyse des Zeitpunktes des RV-Schlusses................26

3.2. Ergebnisse der mikrobiologischen Testung der RVs ..................27

3.2.1. Testung der Verhinderung bakterieller Kontamination durch RVs bei Infusion gegen die RV-Durchflussrichtung.............27

3.2.2. Testung bakterieller Kontamination retrograd über RVs gegen einen Flüssigkeitsstrom in RV-Durchflussrichtung

4. Diskussion........................................................... 32

4.1. Diskussion der physikalischen Testung der RVs.....................32

4.1.1. Diskussion der Methode ....................................32

4.1.1.1. Prüfung der Hypothese und Literaturrecherche..........32

4.1.1.2. Klinische Relevanz des Experiments...................32

4.1.1.3. Versuchsaufbau im Vergleich zur DIN-Norm 58362......34

4.1.1.4. Einfluss von $\mathrm{NaCl} 0.9 \%$-Lösung auf die

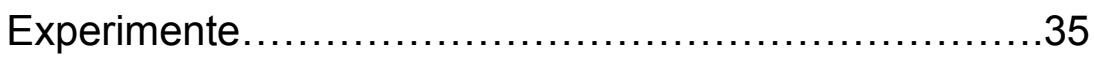

4.1.2. Diskussion der Kurvenverläufe und Steigungsbeginne............35

4.2. Diskussion der mikrobiologischen Testung der RVs...................36

4.2.1. Passage von S. epidermidis durch RVs........................37

4.2.2. Passage von $S$. aureus durch RVs............................ 38

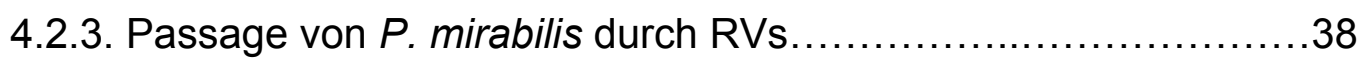


4.3. Limitationen unseres Experiments.....

4.4. Zusammenfassende Beurteilung der RVs.........................41

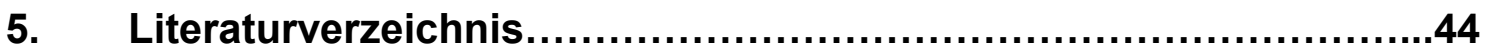

6. Lebenslauf..................................................................

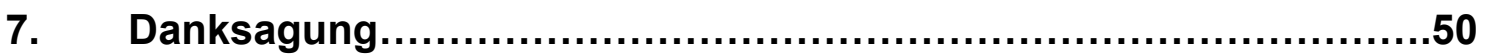

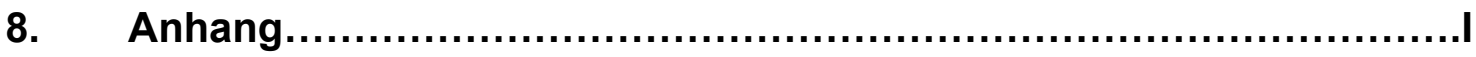




\section{Einleitung und Problemstellung}

\subsection{Funktion von Rückschlagventilen in der Medizin}

Ein Rückschlagventil (RV) ist in der Fluidtechnik ein Wegeventil, welches den Durchgang eines Mediums wie Hydraulikflüssigkeit, Druckluft oder Infusionslösung gegen die Durchflussrichtung sperrt und die Passage in Durchflussrichtung gestattet.

Konstruktiv wird die Sperrung mittels einer Feder ausgelöst, die eine Kugel, einen Kegel oder eine Membran in eine Position bringt, die die Passage gestattet oder den Durchfluss verhindert. Baut sich in Durchflussrichtung ein Druck auf, der größer ist als die Kraft der Feder, wird das dichtende Element vom Sitz abgehoben und der Durchfluss freigegeben.

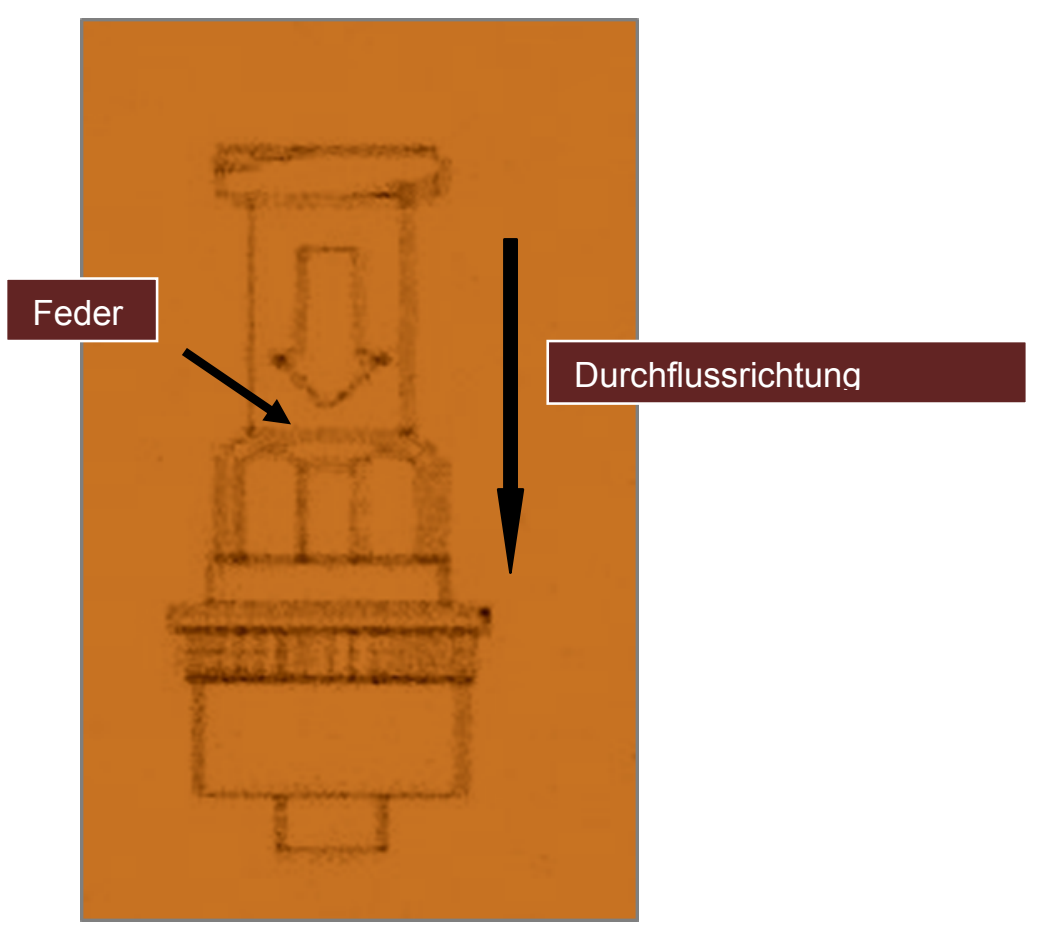

Abbildung 1: $\quad$ Aufbau eines Rückschlagventils (B. Braun Melsungen AG; Rückschlagventil RV-,,Solo“; Artikel-Nummer 4094000N). Gekennzeichnet sind die Durchflussrichtung sowie die Feder, die bei Rückfluss von Infusionslösungen gegen die RV-Durchflussrichtung den Schließmechanismus auslöst und damit den Rückfluss der Infusionslösung verhindern soll. 
In der Medizin finden RVs Anwendung bei der schwerkraft- oder pumpengetriebenen Medikamenteninfusion. Sie sollen das Vermischen unterschiedlicher, parallel über einen venösen Gefäßzugang applizierter Infusionsflüssigkeiten verhindern.

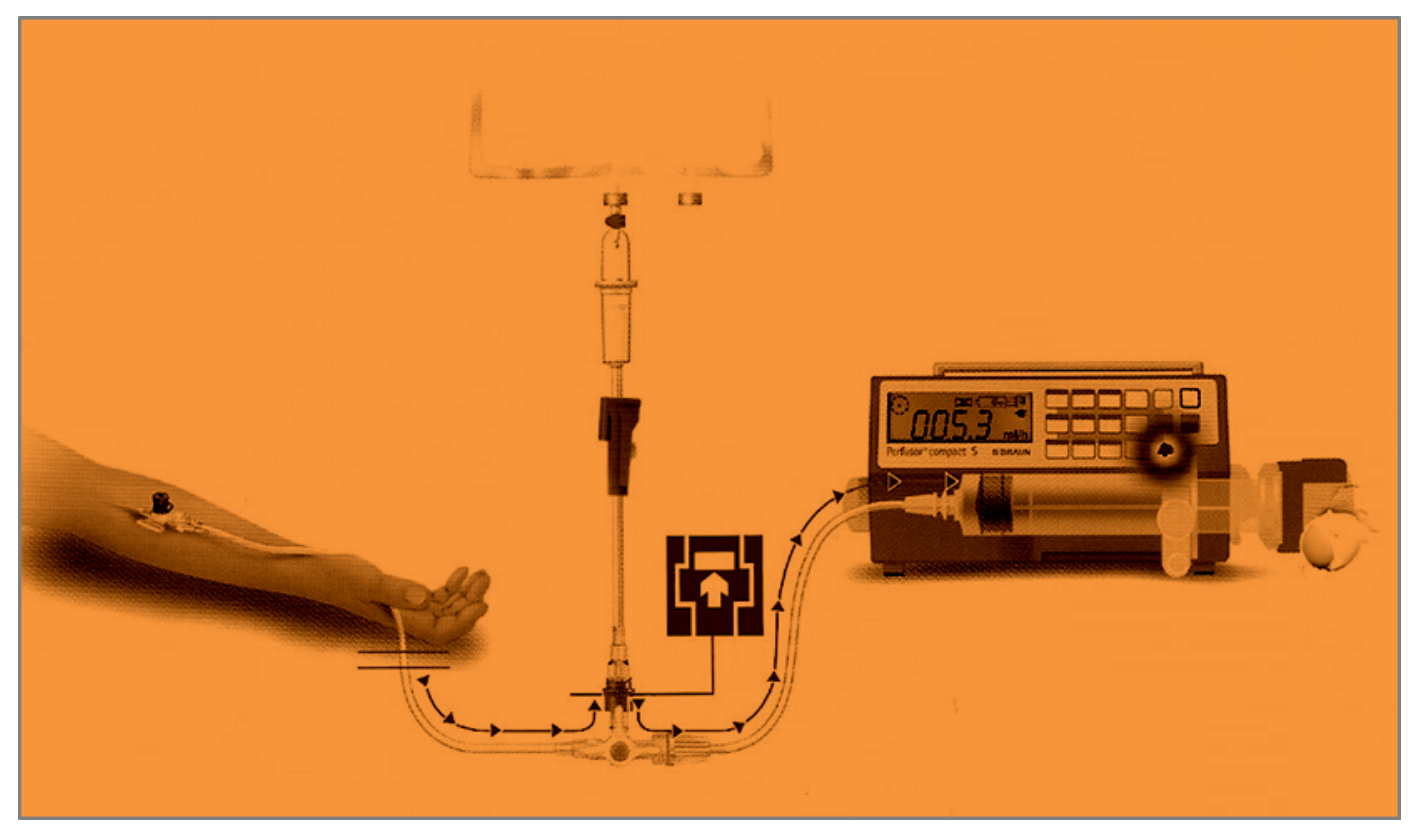

Abbildung 2: $\quad$ Bei Verschluss der Patientenleitung verhindert das RV Rückfluss von Infusionslösung gegen die RV-Durchflussrichtung. Nach Druckaufbau im System wird ein Alarm über die Infusionspumpe ausgelöst (Aus: Detailinformation Rückschlagventile, ArtikelNummer 4094000N; B. Braun Melsungen AG).

Bei Unterbrechung einer Infusion durch ein patientenseitiges Infusionshindernis, z.B. durch Abknickung der Druckleitung, sollen RVs einen Blut- oder Medikamentenfluss in das Schwerkraftinfusionssystem verhindern. Der RVSchliessmechanismus soll unmittelbar einsetzen, wenn ein Fluss gegen die RVDurchflussrichtung aufgebaut wird. Ein konsekutiv im System entstehender Druckanstieg wird von der Infusionspumpe registriert, und ein akustisches Alarmsignal ertönt.

Um einen universellen Gebrauch zu gewährleisten, werden RVs mit DINgenormten Luer-Lock Konnektoren produziert. Durch Kennzeichnung der Durchflussrichtung ermöglichen die Hersteller dem Anwender einen einfachen 
Einbau in Infusionssysteme. Beidseitige Schutzkappen dienen einer kontaminationsgeschützten Handhabung. Ein objektiver Vergleich unterschiedlicher RVs ist schwierig. Detaillierte physikalische Informationen können der Produktinformation nicht entnommen werden.

\subsection{Anforderungen an Rückschlagventile}

\subsubsection{Verhinderung von Rückfluss in das Infusionssystem}

Fehlfunktionen von RVs können im klinischen Gebrauch zu einer Medikamentenfehldosierung führen und damit schwere Folgen für die Patienten haben. Bei RV-Fehlfunktion kann bei parallel applizierten Medikamenten ein Rückstau von Infusionslösung in das Schwerkraftinfusionssystem resultieren. Bei nachfolgend einlaufender Infusion kann es zu einer Medikamentenfehldosierung durch Applikation der zurückgestauten Infusionslösung kommen.

\subsubsection{Allgemeine Qualitätsanforderungen an Rückschlagventile}

An RVs werden unterschiedliche Qualitätsanforderungen gestellt (Hintzenstern, 2004).

-Rückflussverhinderung: $\quad$ RVs müssen bei niedrigster Förderrate der Infusionspumpe $(0,1 \mathrm{ml} / \mathrm{h})$ sicher schließen und einen Infusionslösungsrückfluss gegen die RV-Durchflussrichtung verhindern.

-Minimale Durchflussminderung: Ein RV darf den Fluss der Infusionslösung in RV-Durchflussrichtung nicht beeinträchtigen.

-Hoher Berstdruck: Ein hoher Berstdruck mit einer Druckfestigkeit von 6 bar muss eine Zerstörung des RVs verhindern. 
-Öffnung:

Durch den hydrostatischen Druck der

Schwerkraftinfusion muss sich das RV öffnen.

\subsubsection{Schutz vor Kontamination durch Rückschlagventile}

Häufig sind nach Beendigung einer Operation die Perfusorspritzen mit den Medikamenten, z.B. zur intravenösen Anästhesie (Propofol, Remifentanyl etc.) noch nicht leer. Es besteht der Wunsch, teure Medikamentenreste ggf. nach Wechsel von Teilkomponenten des Infusionssystems (RVs, ggf. inklusive einer Druckleitung) für andere Patienten weiter verwenden zu können. Die verwandten Infusionssysteme ohne RVs sind für dieses Vorgehen nicht zugelassen, da eine Kontamination der Medikamente möglich ist. Bei Anwendung von RVs kann der Anwender annehmen, durch das RV eine bakterielle Kontamination der Infusionslösung zu verhindern. Gemäß Produktinformation der Hersteller von RVs, Infusionssystemen und von Infusionslösungen wie beispielsweise Propofol verbietet sich dieses Vorgehen, auf Grund der Gefahr Bakterien von einem Patienten zu einem nachfolgenden zu übertragen (Astra Zeneca, 2004). Valide Daten hierzu finden sich in der publizierten Literatur nicht. Lessard et al. zeigten, dass es bei Weiterverwendung von Perfusorspritzen zu bakterieller Kontamination kommen kann (Lessard et al. 1988). Nur 1/3 der Kontaminationen sind makroskopisch sichtbar. Okkultes Blut kann Bakterien und auch Viren übertragen (Trepanier et al. 1990). Geringe Mengen von ca. $10^{-9} \mathrm{ml}$ Blut reichen aus, um Hepatitis B, Hepatitis C oder HI-Viren zu übertragen (Hein et al. 1987; Kempen u. Learned 1989; Koepke et al. 1985).

Hersteller von Propofol weisen darauf hin, das Medikament nur für einen einzelnen Patienten zu verwenden. Die Infusion von unverdünntem Propofol 1\% aus einem Infusionssystem darf eine Dauer von 12 Stunden nicht überschreiten. Bei Verwendung von gebrauchsfertigen Mischungen mit 5\%iger Glukoselösung darf diese Infusionslösung maximal 6 Stunden angewendet werden (AstraZeneca 01/2004). 
Das Robert-Koch-Institut (RKI) veröffentlicht Richtlinien für die Krankenhaushygiene. Diese beinhalten Leitlinien für die Verwendung und Handhabung von Infusionssystemen. Die Möglichkeit der Integration von RV in Infusionssysteme findet weder in der Produktinformation noch in den Empfehlungen des RKI Berücksichtigung (Kramer 2002).

\subsection{Studienziel und Hypothese}

Wir stellten die Hypothese auf, das RVs eine Kontamination des Infusionssystems nicht sicher verhindern können.

Ziel unserer Studie war, zu untersuchen, ob RVs einen Rückfluss in eine Schwerkraftinfusion sicher verhindern können. Zudem sollte die Hypothese geprüft werden, ob RVs eine bakterielle Kontamination unterschiedlicher Infusionslösungen ( $\mathrm{NaCl} 0.9 \%$ und Propofol) verhindern können.

Der Arbeitsausschuss B5 „Entnahme- und Übertragungsgeräte“ des Normenausschusses Medizin (NAMed) im Deutschen Institut für Normung e.V. (DIN) hat für die Testung von RVs einen Normenentwurf (58 362-7) erarbeitet. Die Testkriterien liegen dem Aufbau unseres Experimentes zugrunde (DINNorm 58 362-7, 2000).

Im ersten Teil des Experiments wurde das Schließverhalten der RVs durch Messung von Druckanstiegen über die Versuchszeit bei Infusion gegen die RVDurchflussrichtung getestet.

Im zweiten mikrobiologischen Teil wurde getestet:

- ob Bakterien RVs bei Druckaufbau gegen die RV-Durchflussrichtung (geschlossener Zustand des RVs) passieren und Druckleitungen und Infusionslösung kontaminieren können.

- ob Bakterien die RVs auch retrograd gegen einen Flüssigkeitsstrom in RV-Durchflussrichtung (geöffnete RVs) passieren und Druckleitungen und Infusionslösung kontaminieren. 
2. Material und Methoden

2.1. Material und Methoden der physikalischen Testung

2.1.1. Die getesteten Rückschlagventile

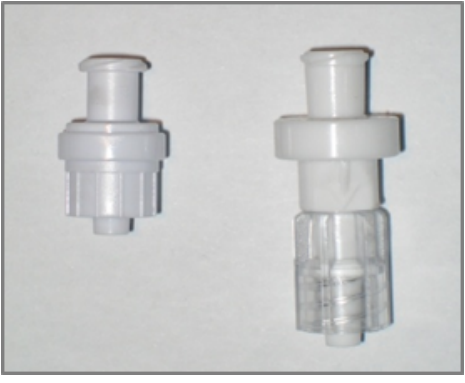

Abbildung 3: Modelle von Smith \& Nephew (re) und Fresenius (li) (Foto aus unserem Experiment)

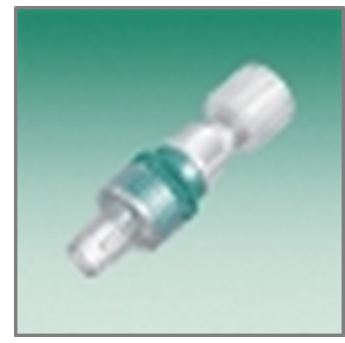

Abbildung 5: Modelle von B. Braun Melsungen AG (Aus: Produktinformation)
Abbildung 4: Modell von Becton, Dickinson and Company (Aus: Produktinformation)

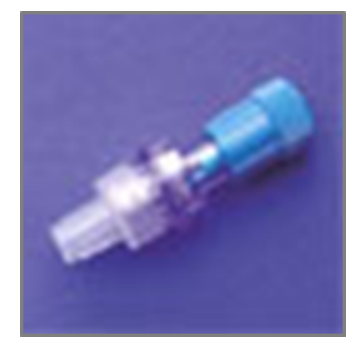

Unsere Experimente wurden an 5 verschiedenen RV-Modellen von 4 Herstellern durchgeführt. In jeder Vergleichsgruppe wurden 40 RVs je Hersteller und Modell getestet. In unserem Experiment wurde gemäß den Standardarbeitsanweisungen zur Prävention Gefäßkatheter-assoziierter Infektionen, erstellt für die Infusionstherapie von der Deutschen Gesellschaft für Krankenhaushygiene e.V. (DGKH), getestet (Kramer 2002). Hierzu gehörten u.a. die hygienische Händedesinfektion, die Wischdesinfektion von Durchstechstopfen und das Nachwischen mit einem sterilen Tupfer. Es wurden sterile, originalverpackte Materialien verwendet. Diskonnektionen wurde auf ein 
Minimum beschränkt. Vor jeder Diskonnektion wurde eine hygienische Händedesinfektion durchgeführt. Katheteransatzstücke bzw. Dreiwegehähne wurden mit Alkohol sprühdesinfiziert.

\subsection{Testung des Schließverhaltens der Rückschlagventile}

\subsubsection{Versuchsaufbau}

Für die Testung des Schließverhaltens der RVs erstellten wir den in Abbildung 6 gezeigten Versuchsaufbau.

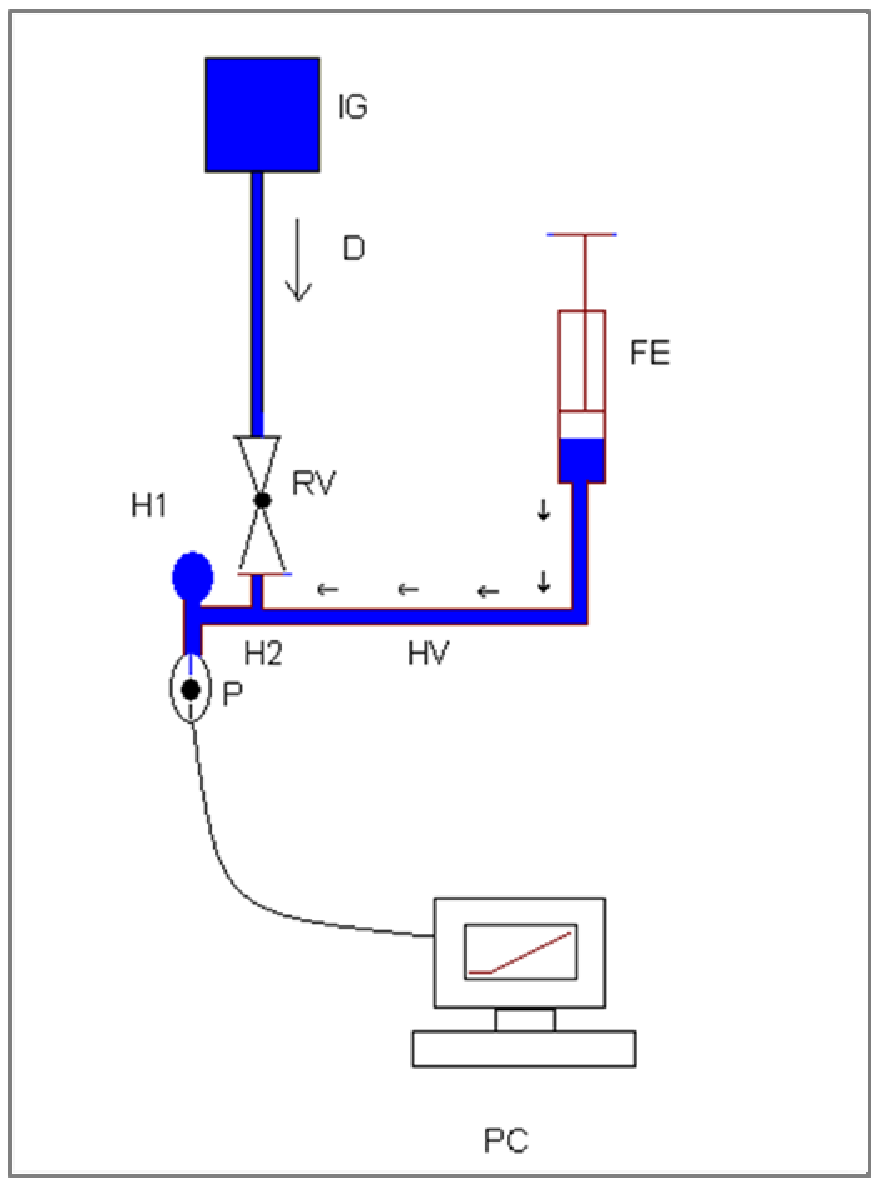

Abbildung 6: $\quad$ Schematische Darstellung des Versuchsaufbaus: Infusionslösung (IG), die klinisch einem zu applizierenden Medikament entspricht. Die pumpengetriebene Infusion FE baute durch Volumenverschiebung einen kontinuierlichen Druck gegen die RVDurchflussrichtung (D) auf. Im klinischen Alltag würde FE einem Patienten entsprechen, der durch Abknickung der Druckleitung oder gleichzeitige Blutdruckmessung einen 
Druck gegen die RV-Durchflussrichtung aufbaut. Die Infusionsleitung (HV), war über

Dreiwegehähne (H1/2) mit einem Druckmessgerät $(P)$ verbunden. Bei

Volumenverschiebung von FE gegen die RV-Durchflussrichtung entstanden nach RV-

Verschluss proportional zum verschobenen Volumen Druckanstiege, die an einem

Druckmessgerät gemessen und auf einem Monitor (PC) gezeigt wurden.

Mit einem durch Verschlussstopfen an der Position des später in den Versuchsaufbau eingebauten RVs abgedichteten System wurden je Perfusor 10 Referenzmessungen durchgeführt. Die arithmetischen Mittelwerte der Messungen waren die Referenzwerte (Siehe S.18/19).

Um Perfusor-bedingte Druckschwankungen zu eliminieren wurden die ZeitDruck-Kurven der getesteten RVs mit der Referenzkurve desselben Perfusors verglichen.

\subsubsection{Versuchsablauf}

Die Druckwerte wurden über die Versuchszeit von 20 Minuten bei einer Perfusorförderrate von $1 \mathrm{ml} / \mathrm{h}$ gegen die RV-Durchflussrichtung bzw. über 2 Stunden bei $0,1 \mathrm{ml} / \mathrm{h}$ registriert. $0,1 \mathrm{ml} / \mathrm{h}$ ist die kleinste Förderrate der Perfusoren. $1 \mathrm{ml} / \mathrm{h}$ wurde vergleichend getestet, um zu testen, ob die RVs bei höherem Gegendruck besser und schneller schließen.

Registrierte Druckwerte über die Versuchszeit wurden tabellarisch erfasst und in Form von Kurven (Abszisse: Versuchszeit; Ordinate: Druckwerte) graphisch dargestellt.

Für jede Testung des RV-Schließverhaltens wurde ein neues RV in das System eingebaut. Um die Druckleitung (HV) zu entlüften wurde zu Versuchsbeginn zunächst der Dreiwegehahn ( $\mathrm{H} 1)$ nach außen geöffnet. Mit einem Bolus aus dem Perfusor IG in RV-Durchflussrichtung wurde eine RV-Blockierung, z.B. durch Luftblasen, ausgeschlossen (Vgl. Abbildung 6).

Nach Kalibrierung des Druckmessers auf einen Nullpunkt wurde über den Perfusor (FE) mit einer Förderrate von $0,1 \mathrm{ml} / \mathrm{h}$ oder $1 \mathrm{ml} / \mathrm{h}$ kontinuierlich ein Volumen gegen die Durchflussrichtung des RVs verschoben. 
RVs werden als undicht gewertet, wenn ihr Schliessmechanismus verspätet eingesetzt hat, erkennbar an einem verzögerten Druckanstieg. Auch wenn Druckschwankungen über die Versuchszeit zu erkennen waren oder trotz weiterer Volumenverschiebung der Druck im System nicht weiter anstieg, galten die RVs als undicht (Vgl. Kapitel 3.1.).

\subsection{Material und Methoden der mikrobiologischen Testung}

\subsubsection{Die getesteten Bakterienstämme}

\subsubsection{S. epidermidis}

Verwendet wurde das biofilmbildende Indikatorbakterium $S$. epidermidis RP62A, welches ein gram-positiver Keim ist, der zur kommensalen Flora der Haut gehört. S. epidermidis gehört in die Gruppe der koagulasenegativen Bakterien und ist ein häufiger Erreger nosokomial erworbener katheterassoziierter Infektionen. Aus Blutkulturen isolierte koagulasenegative Staphylokokken zeigen auf Intensivstationen in bis zu $70 \%$ multiple Antibiotikaresistenzen.

S. epidermidis wurde für unser Experiment gewählt, da dieser Keim mehrschichtige, auf Polymerstrukturen adhärierende, in eine Glycocalix aus Exopolysacchariden eingebettete Biofilme bildet und damit polymere Oberflächen besiedeln kann.

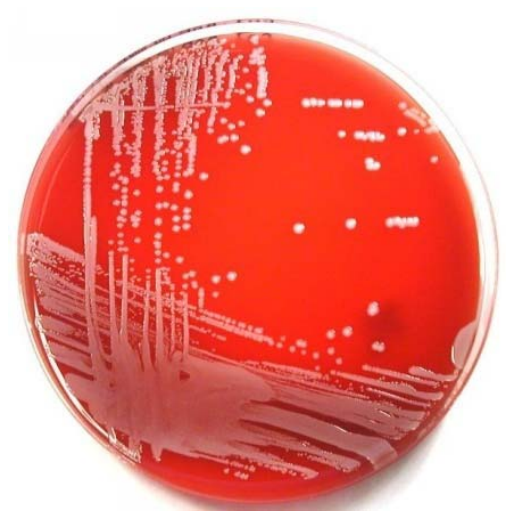

Abbildung 7: $\quad$ S. epidermidis 


\subsubsection{S. aureus}

S. aureus ATCC 25923 ist ein koagulasepositiver Bakterienstamm. Eine Infektion mit diesem Keim kann u.a. Pneumonien, Osteomyelitiden, Endokarditiden, Wundinfektionen oder katheterassoziierter Infektionen induzieren. 18,3\% der häufigsten Infektionen auf Intensivstationen (Harnwegsinfektionen, Pneumonien, Sepsis und Wundinfektionen) werden durch MethicillinResistenten-Staphylococcus-aureus induziert (Robert-Koch-Institut 2007).

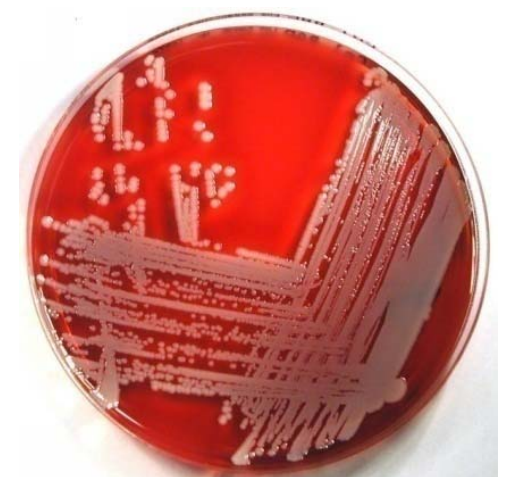

Abbildung 8: $\quad$ S. aureus

\subsubsection{3. $\quad P$. mirabilis}

P. mirabilis ATCC 35659 gehört zur Spezies der Familie der Enterobacteriaceae und zur Gattung Proteus. Hierbei handelt es sich um ein fakultativ pathogenes, anaerobes, gramnegatives, peritrich begeißeltes und damit sehr bewegliches Stäbchenbakterium, welches bei Menschen und Tieren zur residenten Darmflora gehört. Aufgrund einer peritrichen Begeißelung zeigt der Keim ein schwärmendes Wachstum (Schwärmphänomen). Das Schwärmphänomen bezeichnet die Fähigkeit von bestimmten ProteusStämmen, bei eingeschränkter Beweglichkeit der Geißel und hoher Bakteriendichte zu einem Synzytium mit mehreren Kernäquivalenten zu fusionieren (sog. Schwärmer-Form). Dieses Synzytium besitzt eine etwa um den Faktor 500 höhere Geißelexpression und ist damit sehr beweglich. 


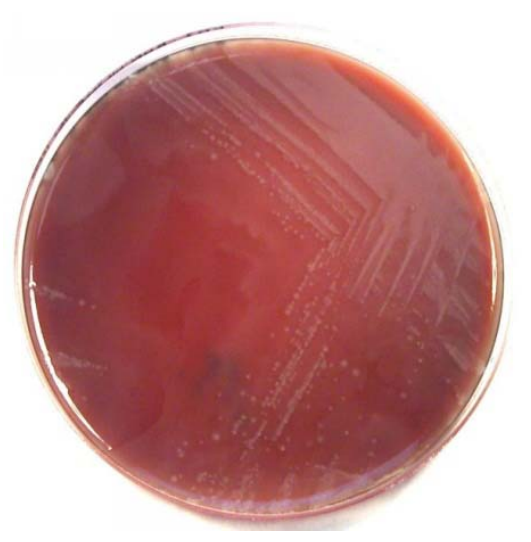

Abbildung 9: P. mirabilis

\subsubsection{Die getesteten Infusionslösungen}

\subsubsection{Propofol (Disoprivan 1\% ®; AstraZeneca GmbH; 01/04)}

Propofol (2, 6-Diisopropylphenol) ist ein Alkylphenolderivat mit der Summenformel $\mathrm{C}_{12} \mathrm{H}_{18} \mathrm{O}$. Aufgrund seiner guten pharmakologischen Eigenschaften (gute Penetration durch die Blut-Hirn-Schranke; schnelles Anund Abfluten) ist das Hypnotikum Propofol in der Anästhesiologie etabliert. Bakterielles Wachstum wird durch die lipidhaltigen Zusammensetzung und wegen des Fehlens antimikrobieller Konservierungsmittel (Arduino et al. 1991; Kuehnert et al. 1997; Sosis et al. 1995; Sosis u. Braverman 1993; Tessler et al. 1992) in Propofol begünstigt. Infusionsflaschen und -systeme sind nur zur einmaligen Anwendung bei einem Patienten zugelassen.

\subsubsection{Natriumchlorid $0.9 \%$ B.Braun}

Isotone Kochsalzlösung $\mathrm{NaCl}$ 0.9\% B.Braun ist eine kristalloide Flüssigkeit, die von Bakterien nicht verstoffwechselt werden kann. $\mathrm{NaCl} 0.9 \%$-Lösung ist vielfach verwendetes Basismedium für die Herstellung von Mischinfusionen oder dient als Vor- oder Nachlauf bei Applikation von Kurzinfusionen. 


\subsubsection{Testung der Verhinderung bakterieller Kontamination durch RVs bei Infusion gegen die RV-Durchflussrichtung}

In diesem Experiment wurden zwei RV-Modelle daraufhin getestet, ob sie bei Volumenverschiebung gegen die RV-Durchflussrichtung eine bakterielle Kontamination von Druckleitungen verhindern können. Das erste RV-Modell gehörte in die Vergleichsgruppe, die im physikalischen Teil am häufigsten eine Volumenverschiebung von Infusionslösung durch RV-Verschluss verhinderte. Das zweite Modell gehörte in die Vergleichsgruppe, die am häufigsten undicht war. Um zu testen, wie häufig Bakterien geschlossene RVs passieren und Druckleitungen kontaminieren konnten, wurden zehn Versuche je Bakterienstamm (S. epidermidis, S. aureus, P. mirabilis) und je RV-Modell bei einer Förderrate von $0,1 \mathrm{ml} / \mathrm{h}$ über eine Versuchszeit von 2 Stunden durchgeführt.

\subsubsection{Versuchsablauf}
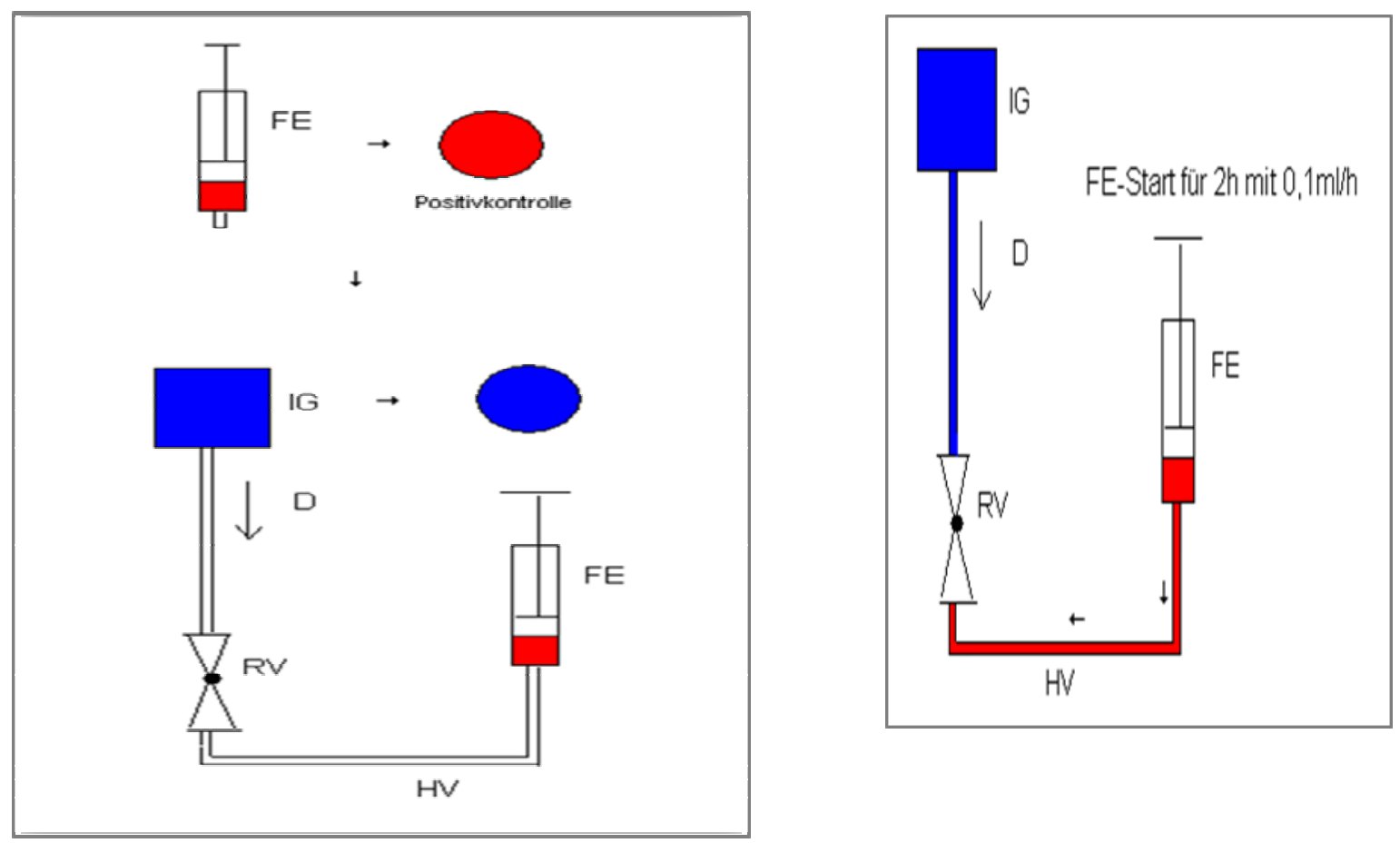
Abbildung 10: Versuchsablauf. Rot= kontaminierte Flüssigkeit. Blau=NaCl 0.9\%. Der Perfusor (FE) baute durch Volumenverschiebung gegen die RV-Durchflussrichtung einen Druck auf. FE wurde mit $\mathrm{NaCl} 0.9 \%$ befüllt und mit einem Bakterienstamm kontaminiert. Unter sterilen Bedingungen wurden Druckleitung (HV), RV sowie der mit $\mathrm{NaCl} 0.9 \%$ gefüllte Perfusor (IG), konnektiert. Zum Ausschluss unbeabsichtigter bakterieller Kontamination Ausstrich von 0,1 ml der Infusionslösung auf Columbia-Blutagarplatten (siehe Abbildung 13) . Mit einer Förderrate von $0,1 \mathrm{ml} / \mathrm{h}$ wurde über 2 Stunden Volumen von (FE) zur Infusionslösung (IG) verschoben.

Um bakterielle Kontamination der Oberfläche auszuschließen wurde nach Ablauf der Versuchszeit (2 Stunden) die Oberfläche des Systems unter sterilen Bedingungen mit 70\%igem Isopropanol desinfiziert. Mit einem in Trypticase Soy Broth-Bouillon (TSB) getränkten Tupfer wurde ein Abstrich vom RV entnommen und kultiviert (siehe Abbildung 14), um nachzuweisen, ob Bakterien das geschlossene RV passieren konnten. Eine weitere Positivkontrolle wurde aus dem Perfusor, der den kontaminiertem Patienten entsprach (FE) entnommen. Kultivierung der Lösung in Bouillon und Ausstrich auf Agar, zur Kontrolle, ob am Versuchsende nur der Stamm enthalten ist, mit dem die Lösung zu Versuchsbeginn kontaminiert wurde.

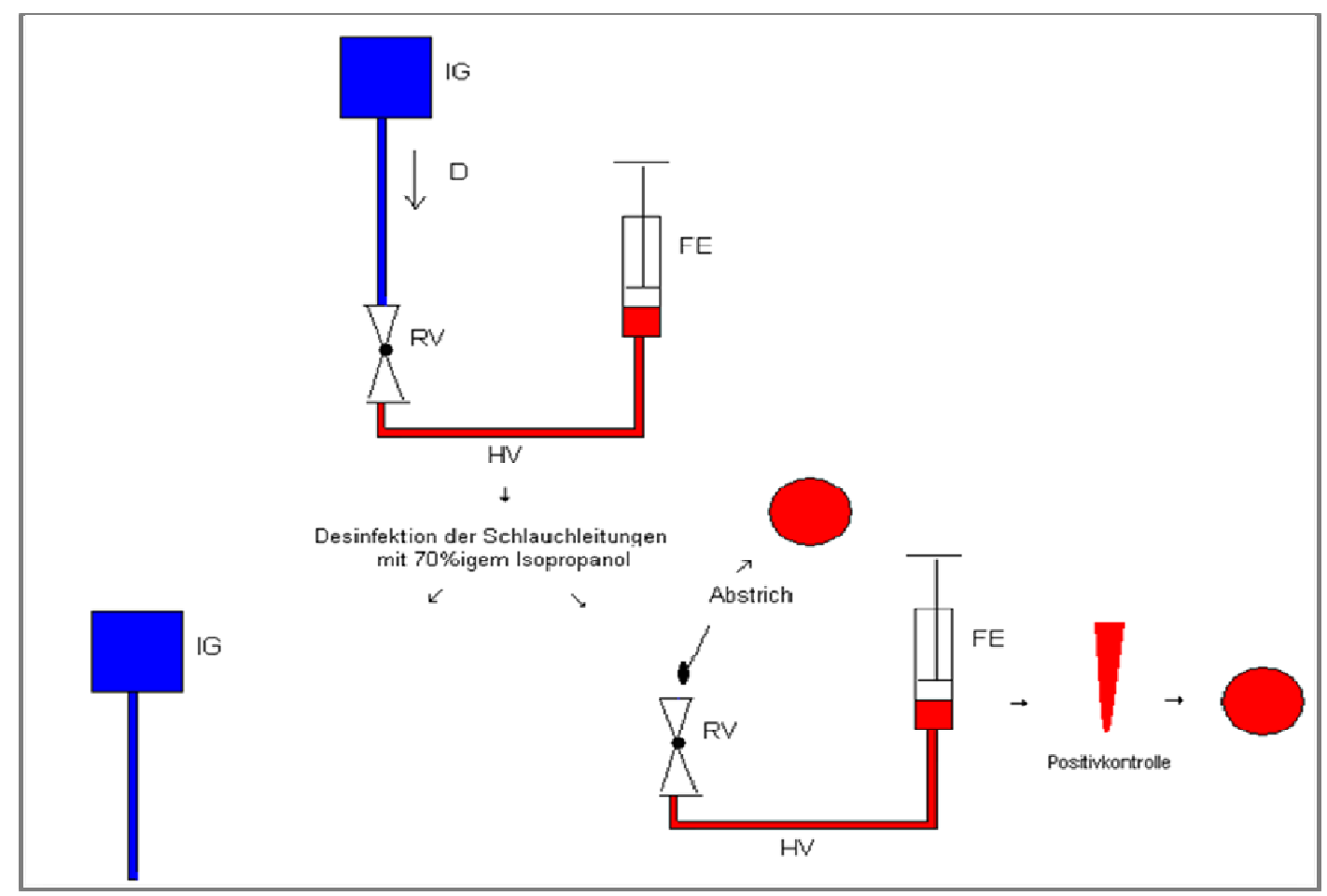

Abbildung 11: $\quad$ RV=Rückschlagventil gegen dessen Durchflussrichtung (D) über den kontaminierten Perfusor (FE) und über eine Druckleitung (HV) ein Druck aufgebaut wurde. 
Unter einer sterilen Werkbank wurde die Druckleitung vom distalen (vom RV entfernten) zum proximalen (RV-nahen) Teil in 11.Teile geschnitten. Jeder Teil der Druckleitung (siehe Abbildung 14) wurde in einem Röhrchen mit Trypticase Soy Broth-Bouillon für 48 Stunden bei $37^{\circ} \mathrm{C}$ bebrütet. Anschließend wurde ein Teil der Lösung auf Columbia-Blutagar ausgestrichen, um zu überprüfen, ob der Bakterienstamm, der sich im System des simulierten Patienten befand, nachweisbar war und somit eine Passage des RVs beweisbar war. Um in unseren Experimenten bei der Testung bakterieller Kontamination falsch positive Ergebnisse durch Bakterien, die am Instrumentarium haften zu verhindern, wurden die Druckleitungen von der Seite aus zerschnitten, die im Versuchsaufbau am weitesten vom RV entfernt war. Durch diese Maßnahme konnte ausgeschlossen werden, dass Bakterien vom Ort hoher Konzentration zum Ort geringerer Konzentration übertragen worden sind.

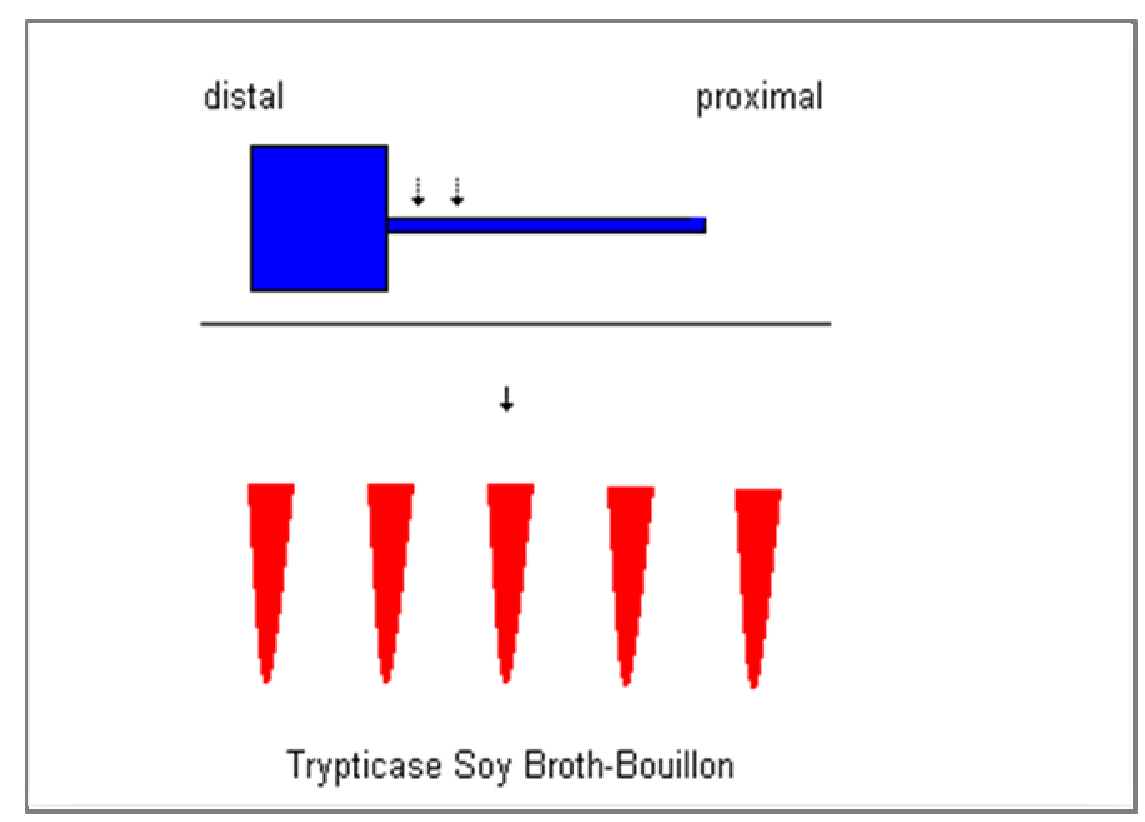

Abbildung 12: Um bakterielle Kontamination auf der Seite nachzuweisen, die mit $\mathrm{NaCl} 0.9 \%$ befüllt war, wurden die Druckleitungen zerschnitten und in Bouillon bebrütet. 

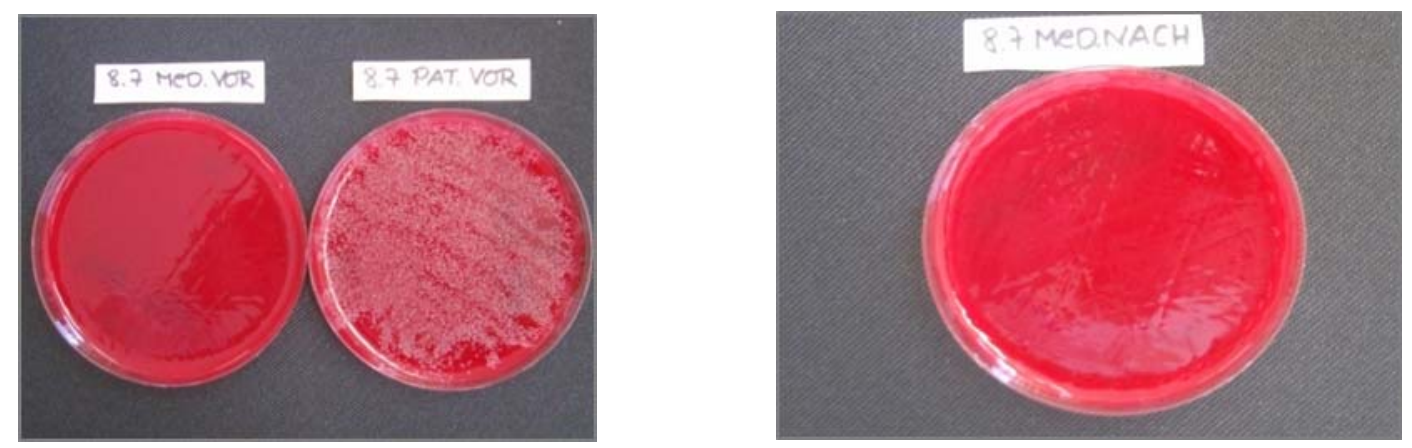

Abbildung 13: Kultivierten Blutagarplatten vor und nach Ablauf der Versuchszeit (Fotos).

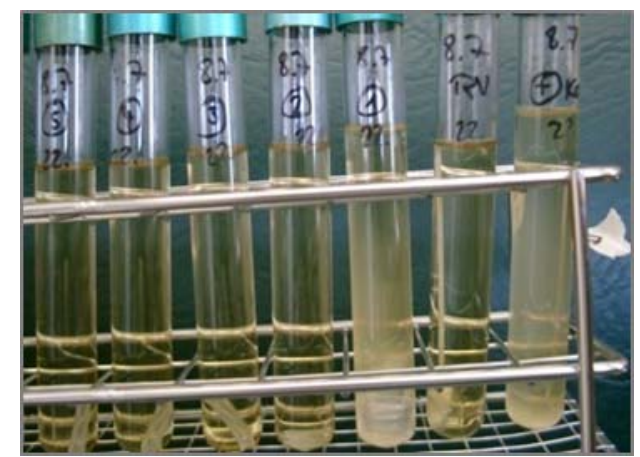

Abbildung 14: $\quad$ Kultivierte Bouillon mit Druckleitungen/RV nach 48 Stunden im Brutschrank $\left(37^{\circ} \mathrm{C}\right)$.

Trübungen bewiesen bakterielle Kontamination (Abbildung 14). Um zu beweisen, dass Trübungen durch zuvor injizierten Bakterienstamm

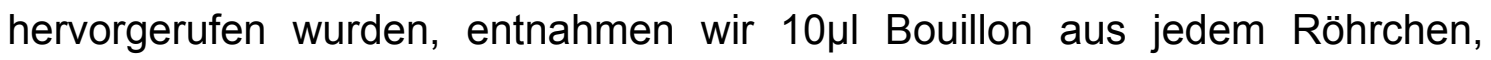
strichen diese mittels 3-Ösenausstrich auf Blutagar aus und bebrüteten diese bei $37^{\circ} \mathrm{C}$ für 48 Stunden.

In tabellarischer Form wurde erfasst, wie häufig bei $n=10$ getesteten RVs pro Bakterienstamm ( $S$. aureus, S. epidermidis, P. mirabilis) eine Kontamination der Druckleitungen über das RV stattgefunden hatte und über welche Strecke (in $\mathrm{cm}$ ) die Druckleitung kontaminiert war. 


\subsubsection{Testung bakterieller Kontamination retrograd über RVs gegen einen Flüssigkeitsstrom in RV-Durchflussrichtung}

\subsubsection{Versuchsablauf}

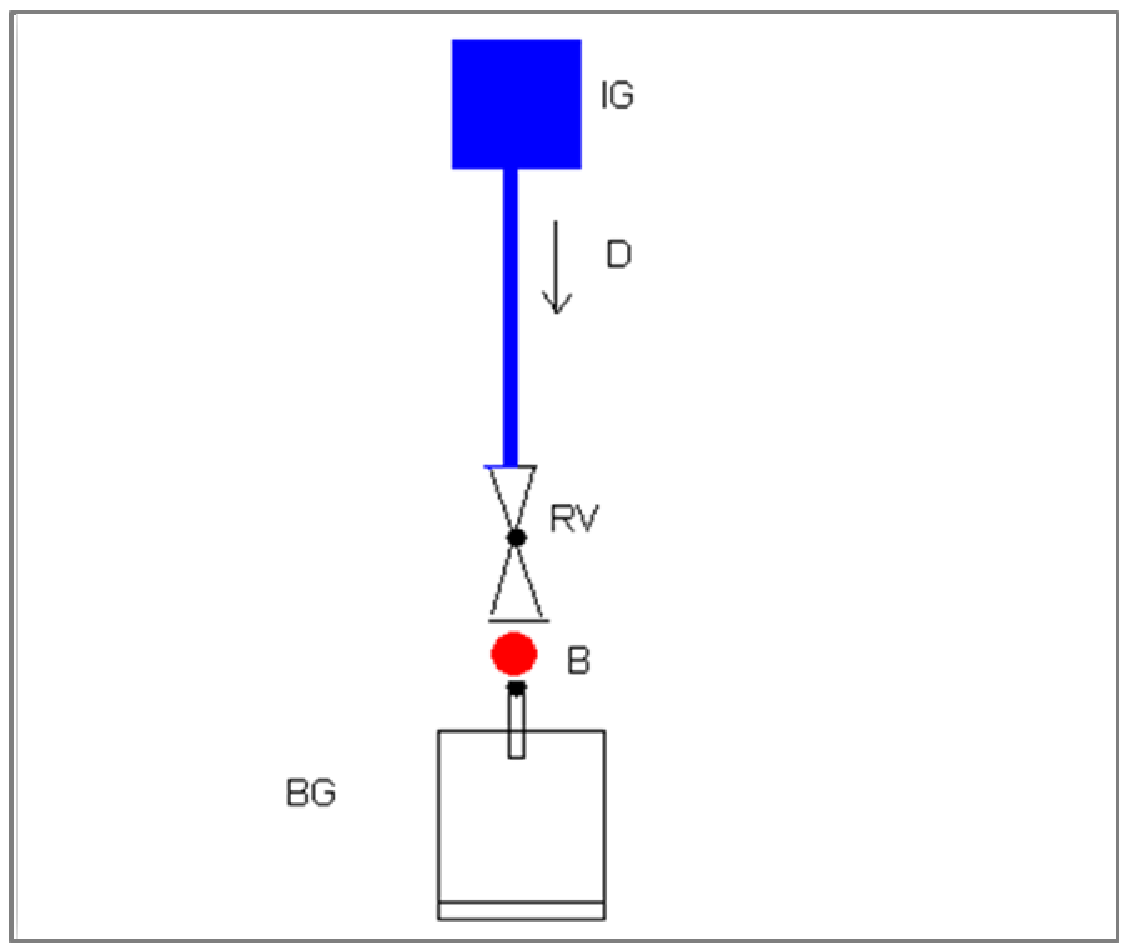

Abbildung 15: Rot=Kontamination. Blau=NaCl 0.9\% oder Propofol. Bakterielle Kontamination (B, rot) an der Seite des RVs, die im klinischen Gebrauch an eine periphere Venenverweilkanüle eines Patienten angeschlossen wäre. Im Versuch wurde die periphere Venenverweilkanüle nach Wischdesinfektion mit 70\%iger Isopropanollösung in ein Becherglas (BG) abgeleitet. BG war mit Parafilm verschlossen. $\mathrm{NaCl} 0.9 \%$ oder Propofol wurden über das Infusionsgerät IG in RV-Durchflussrichtung (D) mit einer Förderrate von $2 \mathrm{ml} / \mathrm{h}$ über 72 Stunden verschoben. IG entspräche klinisch einer Infusionslösung, die einem Patienten (BG) in die RV-Durchflussrichtung appliziert werden soll. Das RV war bei diesem Versuch geöffnet. 


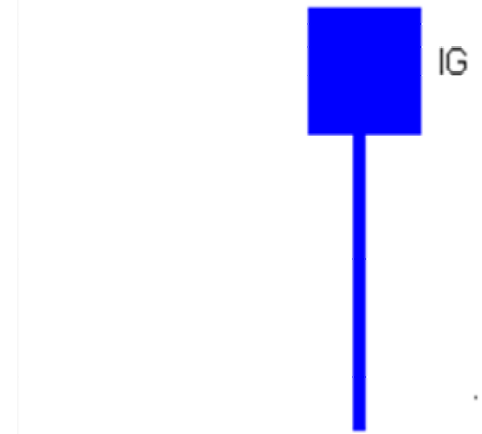

Desinfektion der Schlauchleitungen mit 70\%igem Isopropanol

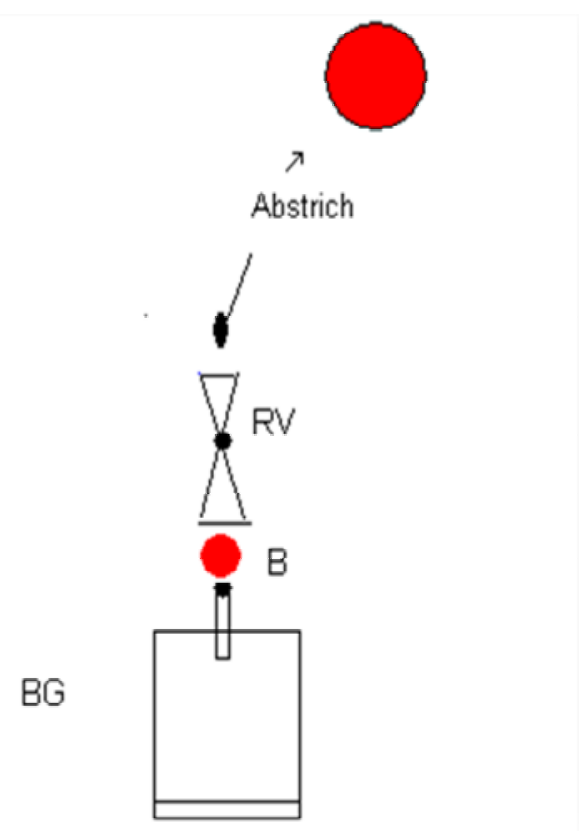

Abbildung 16: $\quad$ Nach 72 Stunden Versuchszeit Desinfektion der Oberfläche der Druckleitungen mit 70\%iger Isopropanollösung. Abstrichentnahme vom RV, auf der Seite, die der Infusionslösung (IG) zugewandt war, um nachzuweisen, ob Bakterien (B) das Druckleitungssystem gegen den Flüssigkeitsstrom in RV-Durchflussrichtung, retrograd kontaminieren konnten.

Zur statistischen Auswertung wurden mit Hilfe von SPSS 14.0 Kreuztabellen erstellt. Bei kleinem Stichprobenumfang wurde zur Berechnung von Signifikanzen ein exakter Test nach Fisher angewendet. Ergebnisse wurden in Balkendiagrammen dargestellt. 


\section{Ergebnisse}

\subsection{Ergebnisse der physikalischen Testung der RVs}

\subsubsection{Referenzmessungen}

Mit jedem Perfusor wurden 10 Referenzmessungen durchgeführt. Bei kontinuierlicher Volumenverschiebung über die Versuchszeit (Min.) stieg der Druck $(\mathrm{mmHg})$ im System proportional an, da über den Stopfen kein Volumen zur Infusionslösung verschoben werden konnte.

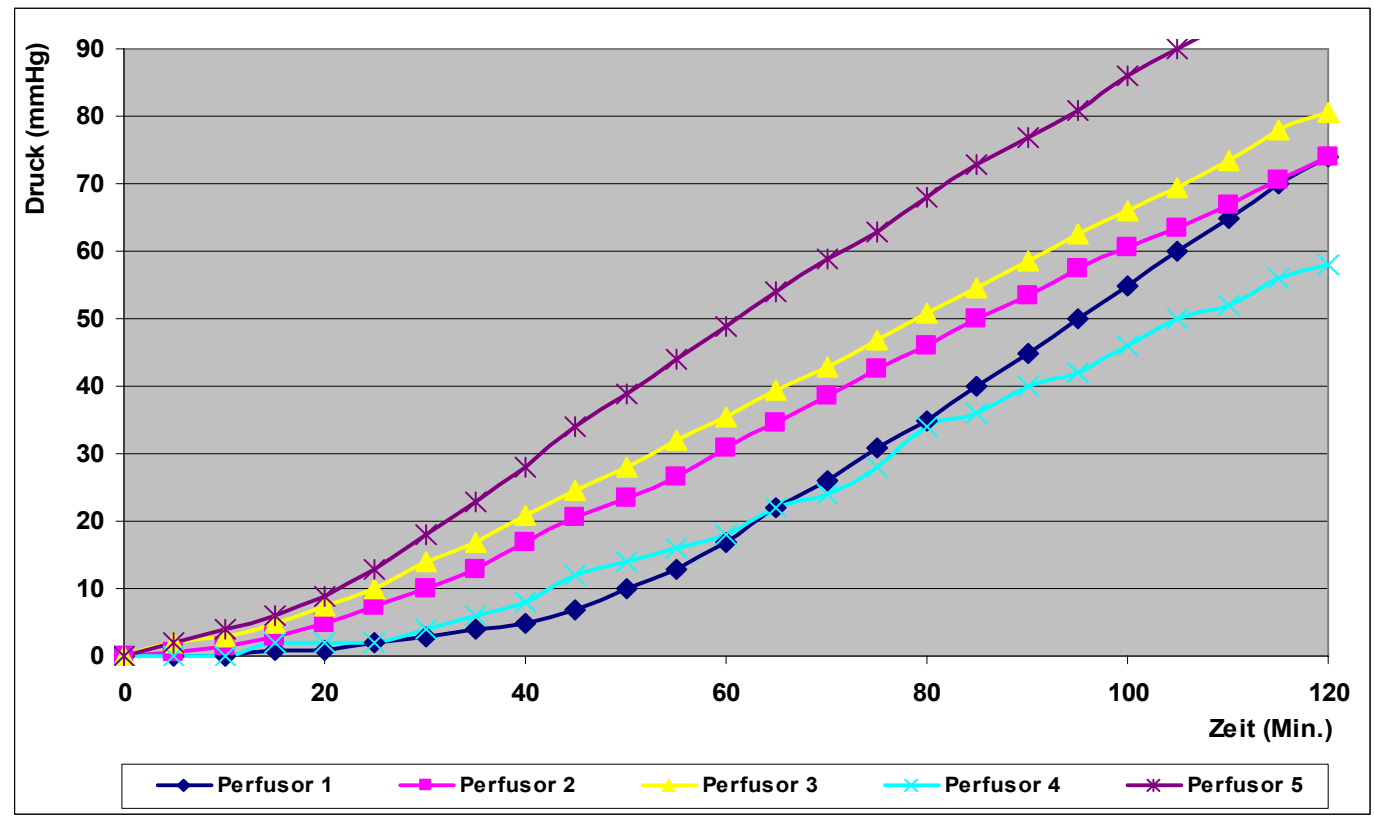

Abbildung 17a: $\quad$ Darstellung der Referenzmessungen. Abgebildet sind die Referenzwerte bei einer Förderrate von 0,1 ml/h über 120 Minuten. 


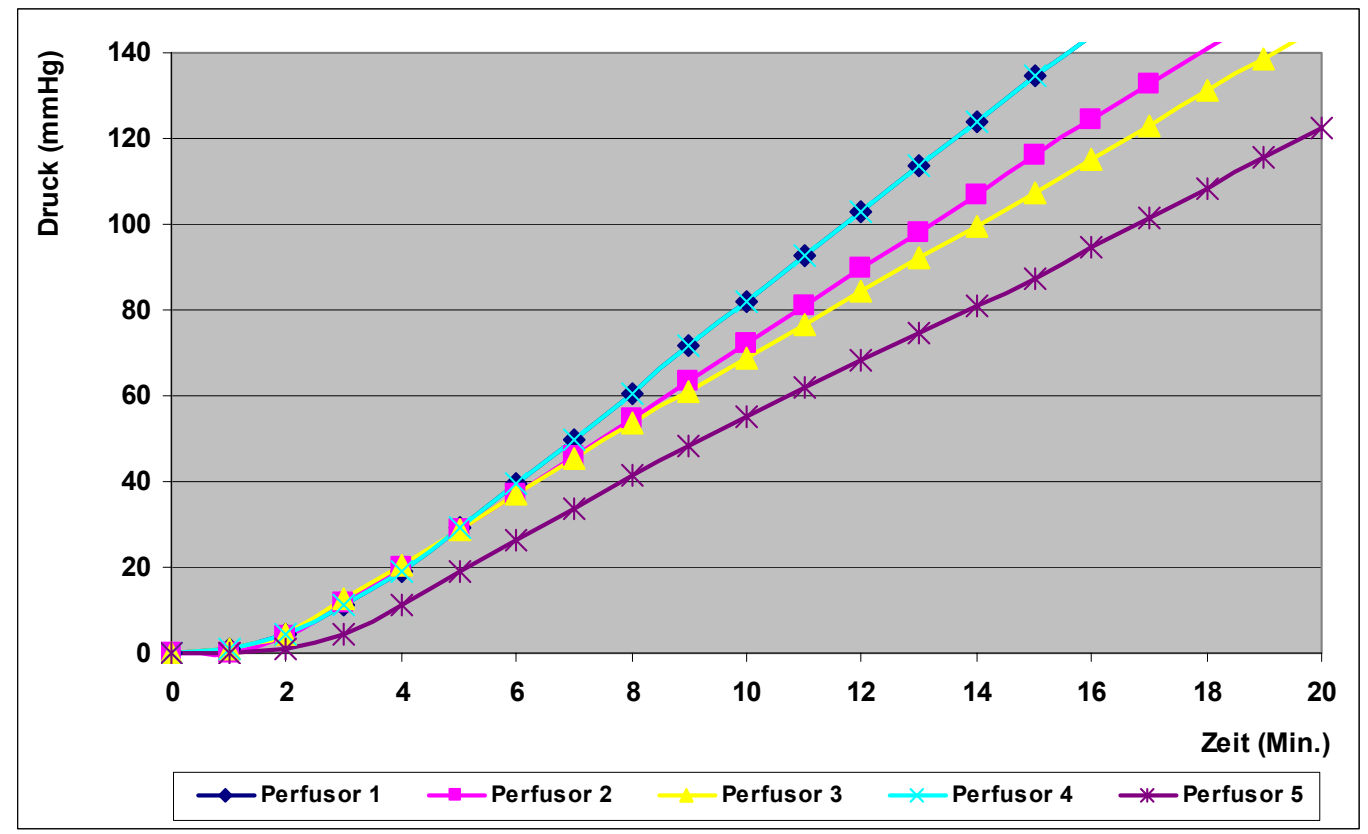

Abbildung 17b: Darstellung der Referenzmessungen. Abgebildet sind die Referenzwerte bei einer Förderrate von $1 \mathrm{ml} / \mathrm{h}$ über 20 Minuten.

\subsubsection{Analyse der Messergebnisse}

Die graphische Darstellung der Ergebnisse (Abbildung 18) mit Zeit-DruckKurven zeigte insgesamt 4 unterschiedliche Kurvenverläufe mit unterschiedlichen, charakteristischen Druckanstiegen über die Zeit:

- „linear" (blau): Druckanstieg bei dichtem RV kontinuierlich, linear und proportional zu dem pro Zeit geförderten Volumen.

- "langsam ansteigend-später linear" (rosa): Nach einer zeitlichen Verzögerung kontinuierlicher, linearer Druckanstieg über die Versuchszeit. Die Verzögerung war bedingt durch Vorlaufzeit der Perfusoren bis effektiv Volumina gefördert werden konnten.

- „unregelmäßig“(gelb): Temporäre Druckabfälle und -anstiege waren zurückzuführen auf Volumenverschiebung über ein undichtes RV.

- "undicht" (grün): Zunächst Druckanstiege, anschließend keine weitere Druckzunahme mehr. Ein einmal erreichtes 
Druckniveau stagniert, da kontinuierlich ein Volumen über ein undichtes RV in Richtung Infusionslösung verschoben wird.
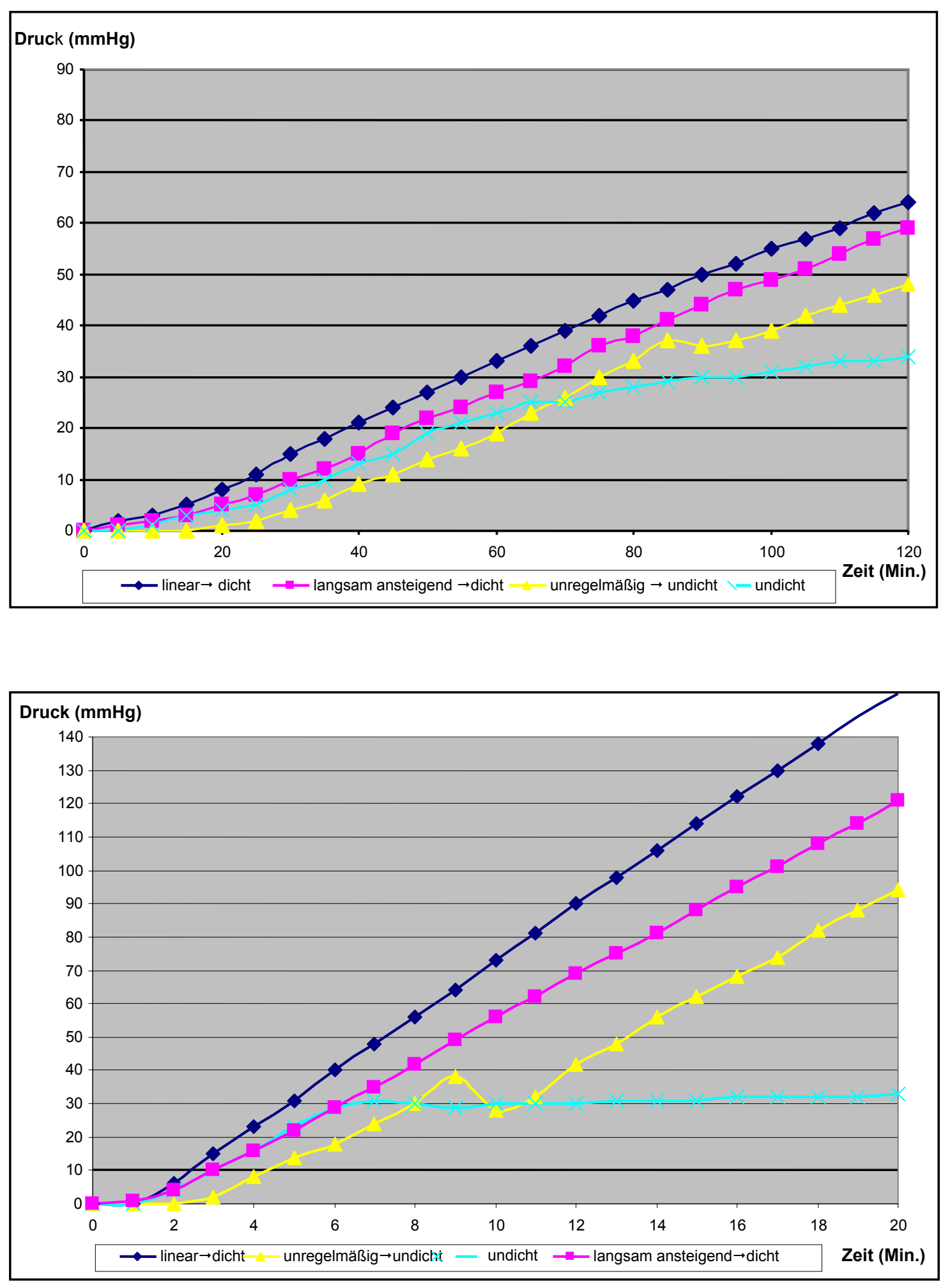
Bei einem dichten Versuchsaufbau ist bei pumpengetriebener Infusion (FE) gegen die RV-Durchflussrichtung über die Versuchszeit ein linearer Druckanstieg am Druckmessgerät abzuleiten, da über den Verschlussstopfen kein Volumen verschoben werden kann (Vgl. Abbildung 6). Jedes verschobene Volumen führt zu einer Drucksteigerung.

Steigungsbeginne nach Start der pumpengetriebenen Infusion, Maximaldruckwerte am Versuchende, die Lage der Kurven im Verhältnis zur Referenzgeraden sowie der Zeitpunkt, von dem an die Kurven linear verlaufen wurden für jedes einzelne RV-Modell ( $n=40$ je Modell) sowie in Summe aller RV-Modelle $(n=200)$ in Tabelle 1 dargestellt.

\begin{tabular}{|c|c|c|c|c|}
\hline Hersteller & Langs.ansteig. & Linear & Unregelmäßig & Undicht \\
\hline RV-Modell-A & $\mathbf{3 3}$ & $\mathbf{7}$ & 0 & 0 \\
\hline RV-Modell-B & $\mathbf{2 3}$ & $\mathbf{3}$ & 12 & 2 \\
\hline RV-Modell-C & $\mathbf{2 8}$ & $\mathbf{4}$ & 3 & 2 \\
\hline RV-Modell-D & $\mathbf{3 2}$ & $\mathbf{6}$ & $\mathbf{4}$ & 0 \\
\hline RV-Modell-E & $\mathbf{2 7}$ & $\mathbf{9}$ & $\mathbf{1 9 = 9 , 5 \%}$ & $9=4,5 \%$ \\
\hline$\Sigma$ & $\mathbf{1 4 3 = 7 1 , 5} \%$ & $\mathbf{2 9 = 1 4 , 5 \%}$ & & \\
\hline
\end{tabular}

\begin{tabular}{|c|c|c|c|c|}
\hline Hersteller & Langs.ansteig. & Linear & Unregelmäßig & Undicht \\
\hline RV-Modell-A & $\mathbf{1 7}$ & $\mathbf{2 2}$ & 0 & 1 \\
\hline RV-Modell-B & $\mathbf{1 7}$ & $\mathbf{2 3}$ & 0 & 0 \\
\hline RV-Modell-C & $\mathbf{2 4}$ & $\mathbf{1 0}$ & 0 & 1 \\
\hline RV-Modell-D & $\mathbf{2 7}$ & $\mathbf{1 2}$ & 0 & 4 \\
\hline RV-Modell-E & $\mathbf{2 4}$ & $\mathbf{9}$ & 3 & $12=6 \%$ \\
\hline$\Sigma$ & $\mathbf{1 0 9 = 5 4 , 5 \%}$ & $\mathbf{7 6 = 3 8 \%} \% 1,5 \%$ & \\
\hline
\end{tabular}


Unabhängig von der Förderrate zeigten sich bei allen RV-Modellen undichte $\mathrm{RVs}$, die eine Volumenverschiebung gegen die RV-Durchflussrichtung nicht verhinderten. RV-Fehlerrate bei einer Volumenverschiebung von $0,1 \mathrm{ml} / \mathrm{h}$ gegen die RV- Durchflussrichtung 14\%. Bei einer Förderrate von $1 \mathrm{ml} / \mathrm{h} 7.5 \%$.

\subsubsection{Analyse der Steigungsbeginne (Minuten) der Kurven}

Nach einer variablen Verzögerungszeit (Förderrate 0,1 ml/h: 5 Min. bis zu $>30$ Min.; Förderrate $1 \mathrm{ml} / \mathrm{h}$ : $1 \mathrm{Min}$. bis zu 4 Min.) nach Start der Infusion, in der noch keine effektiven Volumina verschoben wurden, stieg der Druck im System bei dichtem RV proportional zum verschobenen Volumen an. Ein früher Steigungsbeginn bei einem geschlossenen RV induzierte Kurven mit hohen Maximaldrücken. Eine höhere Förderrate führte zu schnelleren Druckanstiegen im System. Unterschiedliche RV-Modelle zeigten ein unterschiedlich schnelles Ansprechverhalten.

\begin{tabular}{|c|c|c|c|c|c|c|c|}
\hline Zeit (Min.) & $<\mathbf{5}$ Min. & $\mathbf{5}$ Min. & $\mathbf{1 0}$ Min. & $\mathbf{1 5}$ Min. & $\mathbf{2 0}$ Min. & $\mathbf{2 5}$ Min. & $\mathbf{2 3 0}$ Min. \\
\hline RV-Modell-A & 0 & $\mathbf{3 0}$ & $\mathbf{4}$ & $\mathbf{2}$ & 1 & 1 & 2 \\
\hline RV-Modell-B & 0 & $\mathbf{6}$ & $\mathbf{4}$ & $\mathbf{4}$ & 3 & 8 & 15 \\
\hline RV-Modell-C & 0 & $\mathbf{8}$ & $\mathbf{6}$ & $\mathbf{8}$ & 8 & 3 & 7 \\
\hline RV-Modell-D & 0 & $\mathbf{2 8}$ & $\mathbf{6}$ & $\mathbf{0}$ & 2 & 1 & 3 \\
\hline RV-Modell-E & 0 & $\mathbf{2 2}$ & $\mathbf{6}$ & $\mathbf{2}$ & 3 & 2 & 5 \\
\hline$\Sigma$ & $0=0$ & $\mathbf{9 4 = 4 7} \%$ & $\mathbf{2 6 = 1 3} \%$ & $\mathbf{1 6 = 8} \%$ & $17=8,5 \%$ & $15=7,5 \%$ & $32=16 \%$ \\
\hline
\end{tabular}

\begin{tabular}{|c|c|c|c|c|c|}
\hline Zeit (Min.) & $\mathbf{1}$ Min. & $\mathbf{2}$ Min. & $\mathbf{3}$ Min. & $\mathbf{4}$ Min. & $\geq \mathbf{5}$ Min. \\
\hline RV-Modell-A & $\mathbf{2 0}$ & $\mathbf{1 9}$ & 1 & 0 & 0 \\
\hline RV-Modell-B & $\mathbf{6}$ & $\mathbf{2 9}$ & 5 & 0 & 0 \\
\hline RV-Modell-C & $\mathbf{1 3}$ & $\mathbf{2 2}$ & 5 & 0 & 0 \\
\hline RV-Modell-D & $\mathbf{1 3}$ & $\mathbf{2 4}$ & 3 & 0 & 0 \\
\hline RV-Modell-E & $\mathbf{7}$ & $\mathbf{2 8}$ & 4 & 1 & 0 \\
\hline$\Sigma$ & $\mathbf{5 9 = 2 9 , 5 \%}$ & $\mathbf{1 2 2 = 6 1} \%$ & $18=9 \%$ & $1=0,5 \%$ & $0=0 \%$ \\
\hline
\end{tabular}



genannten Zeitintervalle Steigungsbeginne der Kurven. Fett hinterlegte Angaben im Referenzbereich. Die Summenzeile enthält die relative Häufigkeit der Steigungsbeginne nach Start der Volumenverschiebung durch die pumpengetriebene Infusion bei insgesamt 200 gemessenen RVs unabhängig vom Hersteller.

Bei einer Förderrate von $0,1 \mathrm{ml} / \mathrm{h}$ lagen die Druckanstiege im Mittel zwischen 824 Minuten (A: 8,3 Min.; B: 24,9 Min.; C: 17,5 Min.; D: 9,1 Min.; E: 11,9 Min.). Bei einer Förderrate von $1 \mathrm{ml} / \mathrm{h}$ im Mittel Schluss der RVs nach 1-2 Minuten (A: 1,5 Min.; B: 2,0 Min.; C: 1,9 Min.; D: 1,8 Min.; E: 2 Min.). Bei 32 \% der RVs kam es bei einer Förderrate von $0,1 \mathrm{ml} / \mathrm{h}$ zu verspäteten Druckanstiegen. Bei einer Förderrate von $1 \mathrm{ml} / \mathrm{h}$ zeigte sich dies bei $9,5 \%$ der Messungen.

\subsubsection{Analyse der Maximaldrücke $(\mathrm{mmHg})$ am Versuchsende}

Bei einer pumpengetriebene Infusion mit einer Förderrate von $0,1 \mathrm{ml} / \mathrm{h}$ bzw. 1 $\mathrm{ml} / \mathrm{h}$ gegen die RV-Durchflussrichtung wurden am Versuchsende unterschiedliche Maximaldrücke $(\mathrm{mmHg})$ erreicht. Je später der Steigungsbeginn des Drucks durch Volumenverschiebung gegen die RVDurchflussrichtung induziert wurde, und je geringer die Steigung insgesamt war, umso geringer waren die maximalen Druckwerte nach Ablauf der Versuchszeit. 71 (35,5\%) von 200 gemessenen RVs zeigten Maximaldrücke, die kleiner waren als die der Referenzmessungen.

\begin{tabular}{|c|c|c|c|c|c|c|}
\hline $\mathbf{m m H g}$ & $<\mathbf{4 0}$ & $\mathbf{4 0} \leq \mathrm{x}<\mathbf{5 0}$ & $\mathbf{5 0} \leq \mathrm{x}<\mathbf{6 0}$ & $\mathbf{6 0} \leq \mathrm{x}<\mathbf{7 0}$ & $\mathbf{7 0} \leq \mathrm{x}<\mathbf{8 0}$ & $\geq \mathbf{8 0}$ \\
\hline RV-Modell A & 2 & 5 & $\mathbf{9}$ & $\mathbf{1 1}$ & $\mathbf{5}$ & $\mathbf{8}$ \\
\hline RV-Modell B & 16 & 13 & $\mathbf{1 0}$ & $\mathbf{1}$ & $\mathbf{0}$ & $\mathbf{0}$ \\
\hline RV-Modell C & 6 & 8 & $\mathbf{5}$ & $\mathbf{1 0}$ & $\mathbf{5}$ & $\mathbf{6}$ \\
\hline RV-Modell D & 2 & 12 & $\mathbf{1 3}$ & $\mathbf{6}$ & $\mathbf{4}$ & $\mathbf{3}$ \\
\hline RV-Modell E & 2 & 5 & $\mathbf{1 1}$ & $\mathbf{1 3}$ & $\mathbf{6}$ & $\mathbf{3}$ \\
\hline$\Sigma$ & $28=14 \%$ & $43=21,5 \%$ & $\mathbf{4 8 = 2 4 \%}$ & $\mathbf{4 1 = 2 0 , 5 \%}$ & $\mathbf{2 0 = 1 0 \%}$ & $\mathbf{2 0 = 1 0} \%$ \\
\hline
\end{tabular}




\begin{tabular}{|c|c|c|c|c|c|c|}
\hline $\mathrm{mmHg}$ & $<70$ & $70 \leq x<90$ & $90 \leq x<110$ & $110 \leq x<130$ & $130 \leq x<150$ & $\geq 150$ \\
\hline RV-Modell A & 1 & 1 & 8 & 7 & 11 & 12 \\
\hline RV-Modell B & 0 & 3 & 2 & 6 & 16 & 13 \\
\hline RV-Modell C & 3 & 6 & 9 & 18 & 3 & 1 \\
\hline RV-Modell D & 1 & 9 & 6 & 9 & 9 & 6 \\
\hline RV-Modell E & 3 & 3 & 5 & 13 & 15 & 1 \\
\hline$\Sigma$ & $8=4 \%$ & $22=11 \%$ & $30=15 \%$ & $53=26,5 \%$ & $54=27 \%$ & $33=16,5 \%$ \\
\hline
\end{tabular}

Tabelle 3: $\quad$ Maximaldrucke am Versuchsende. Oben Förderrate $0,1 \mathrm{ml} / \mathrm{h}$, unten $1 \mathrm{ml} / \mathrm{h}$. Fett hinterlegte $\sum$-Angaben liegen innerhalb des Referenzbereichs. Die Summenzeile enthält die relative Häufigkeit der maximal erreichten Druckwerte am Versuchsende bei insgesamt 200 gemessenen RVs unabhängig vom Hersteller

\subsubsection{Analyse der Lage der Kurven im Vergleich zur Referenzgeraden}

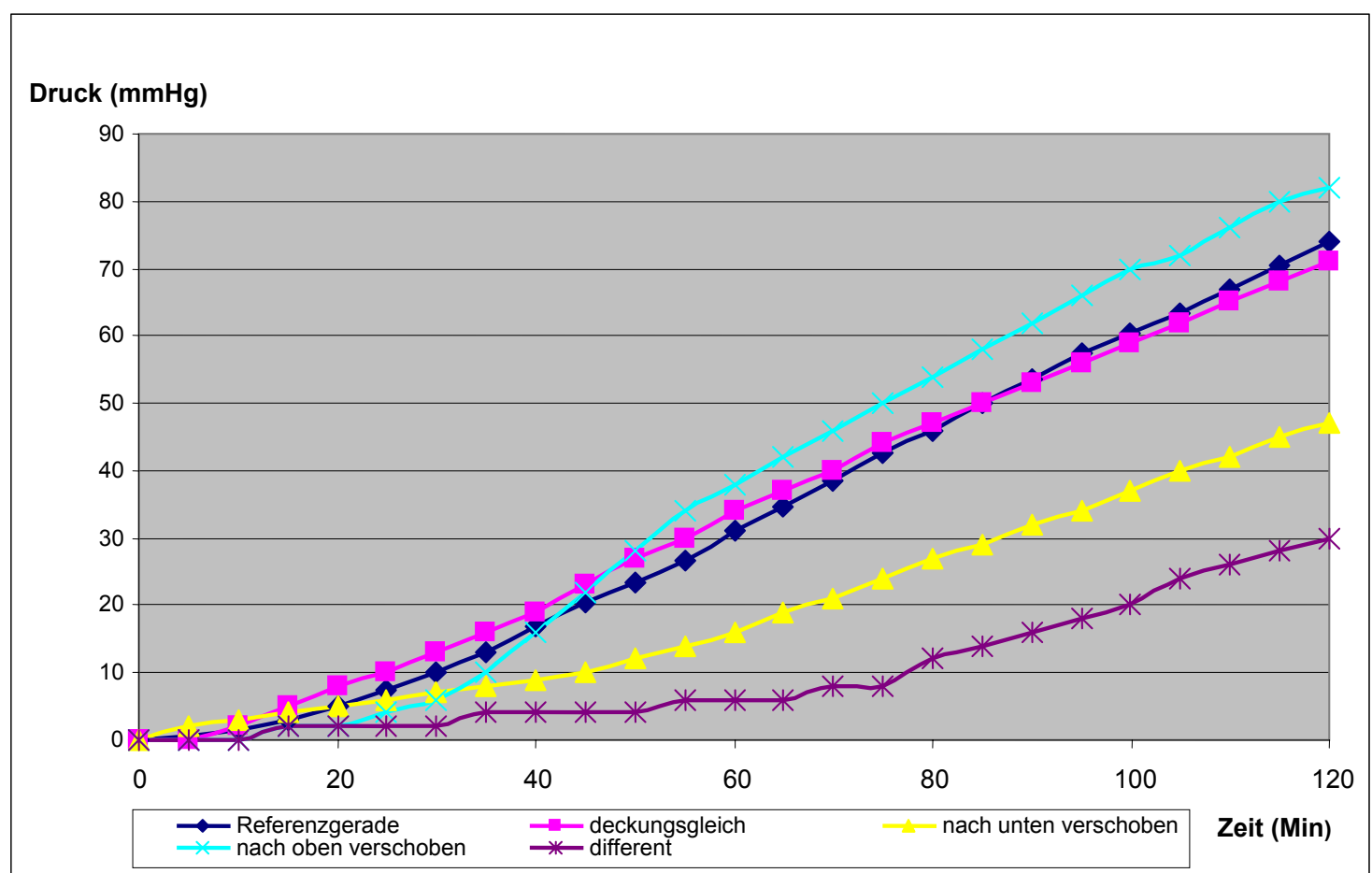

Abbildung 19: Verglichen mit der Referenzkurve (blau) zeigten sich bei pumpengetriebener Infusion gegen die RV-Durchflussrichtung unterschiedliche Kurvenverläufe. Einige Kurven verliefen im Vergleich zur Referenzgeraden nach oben (grün) oder unten (gelb) verschobenen. Andere Verläufe waren deckungsgleichen (rosa) oder komplett different (lila). 
Bei dichtem RV waren Referenzkurve und Kurve der Messung deckungsgleich. Je höher die extern erzeugte Förderrate, umso zuverlässiger setzte der Schließmechanismus der RVs ein.

Insgesamt betrug die Rate an RVs die effektiv eine Volumenverschiebungen gegen die Durchflussrichtung verhindern bei einer Förderrate von $0,1 \mathrm{ml} / \mathrm{h}$ 23,5\% (47 RVs) und bei dem Förderrate von $1 \mathrm{ml} / \mathrm{h} \mathrm{40 \%} \mathrm{(80} \mathrm{RVs).}$

Bei RVs, die eine Druckkurve mit einem nach unten verschobenen Verlauf zeigten, war eine RV-Undichtigkeit nicht sicher auszuschließen (Förderrate 0,1 $\mathrm{ml} / \mathrm{h}: 115 \mathrm{RVs}=57,5 \%$; Förderrate $1 \mathrm{ml} / \mathrm{h}: 106 \mathrm{RVs}=53 \%$ ). Zu späte Steigungsbeginne und zu geringe Maximaldrücke der Kurven am Versuchsende waren bedingt durch eine RV-Undichtigkeit v.a. bei Start der Infusionspumpe. Da diese Kurven nach einer Verzögerungszeit, die durch die Infusionspumpen bedingt war, einen linearen Verlauf aufzeigten, wurden die RVs in unserem Experiment als dichte RVs gewertet. Druckabfälle waren bedingt durch undichte RVs. Die Flüssigkeit wurde über das undichte RV in Richtung des geringsten Widerstands verschoben.

\begin{tabular}{|c|c|c|c|c|}
\hline $\begin{array}{c}\text { Lage im } \\
\text { Vergleich zur } \\
\text { Referenzgeraden }\end{array}$ & $\begin{array}{c}\text { Nach oben } \\
\text { verschobener } \\
\text { Kurvenverlauf }\end{array}$ & $\begin{array}{c}\text { Nach unten } \\
\text { verschobener } \\
\text { Kurvenverlauf }\end{array}$ & $\begin{array}{c}\text { Deckungsgleicher } \\
\text { Kurvenverlauf }\end{array}$ & $\begin{array}{c}\text { Differenter } \\
\text { Kurvenverlauf }\end{array}$ \\
\hline RV-Modell A & 7 & 21 & 8 & 4 \\
\hline RV-Modell B & 0 & 24 & 7 & 11 \\
\hline RV-Modell C & 3 & 19 & 6 & 2 \\
\hline RV-Modell D & 2 & 30 & 12 & 5 \\
\hline RV-Modell E & 2 & 21 & $33=16,5 \%$ & $38=19 \%$ \\
\hline$\sum$ aller Modelle & $14=7 \%$ & $115=57,5 \%$ & 0 & 16 \\
\hline
\end{tabular}

\begin{tabular}{|c|c|c|c|c|}
\hline $\begin{array}{c}\text { Lage im } \\
\text { Vergleich zur } \\
\text { Referenzgeraden }\end{array}$ & $\begin{array}{c}\text { Nach oben } \\
\text { verschobener } \\
\text { Kurvenverlauf }\end{array}$ & $\begin{array}{c}\text { Nach unten } \\
\text { verschobener } \\
\text { Kurvenverlauf }\end{array}$ & $\begin{array}{c}\text { Deckungsgleicher } \\
\text { Kurvenverlauf }\end{array}$ & $\begin{array}{c}\text { Differenter } \\
\text { Kurvenverlauf }\end{array}$ \\
\hline RV-Modell A & 0 & 20 & 19 & 1 \\
\hline RV-Modell B & 2 & 18 & 10 & 4 \\
\hline RV-Modell C & 0 & 26 & 10 & 7 \\
\hline RV-Modell D & 3 & 25 & 13 & $14=7 \%$ \\
\hline RV-Modell E & 3 & 17 & $72=36 \%$ & 2 \\
\hline$\sum$ aller Modelle & $8=4 \%$ & $106=53 \%$ & & 7 \\
\hline
\end{tabular}


Bei Vergleich der Kurvenverläufe der RV-Messungen mit den Referenzmessungen (siehe Tabelle 4) zeigen sich 4 verschiedene Kurvenverläufe. Bei dichten RVs zeigten sich im Vergleich zur Referenzgeraden nach oben, zu höheren Druckwerten verschobene Kurven oder deckungsgleiche Verläufe. Hier wurde kein Volumen über das RV verschoben. Der Druck stieg proportional zur pumpengetriebenen Infusion an. Im Vergleich zur Referenzkurve differente Verläufe zeigten intermittierende Druckabfälle. Über ein undichtes RV konnte Volumen gegen die RV-Durchflussrichtung verschoben werden. Bei einer Förderrate von $0,1 \mathrm{ml} / \mathrm{h}$ gegen die RVDurchflussrichtung betrug die Fehlerrate der RVs 19\% (1 ml/h 9\%). Lediglich das RV-Modell B zeigte bei einer Förderrate von $1 \mathrm{ml} / \mathrm{h}$ keine Messungen mit differenten Verläufen. RV-Modell B zeigte aber die häufigsten, undichten Verläufe bei einer Förderrate von 0,1 ml/h. Verglichen mit der Referenzgeraden parallel nach unten verschobene Kurvenverläufe waren bedingt durch den Mechanismus der pumpenbetriebenen Infusion. Erst nach einer Vorlaufzeit stabilisieren sich die Förderraten der Perfusoren und Förderraten können konstant über die Zeit verschoben werden.

\subsubsection{Analyse des Zeitpunktes des RV-Schlusses}

Nach einer Verzögerungszeit der Perfusoren (Vgl. Trompetenkurven) wurde Volumen durch die Perfusoren gefördert. Die Kurven zeigten Druckanstiege. Der Zeitpunkt, von dem an die Kurven lineare Verläufe aufwiesen entsprach dem Zeitpunkt des RV-Schlusses. 


\begin{tabular}{|c|c|c|c|c|c|c|c|}
\hline Zeit (Min.) & $<10$ & $\begin{array}{c}10 \leq x< \\
20\end{array}$ & $\begin{array}{c}20 \leq x< \\
30\end{array}$ & $30 \leq x<40$ & $40 \leq x<50$ & $\geq 50$ & k.A. \\
\hline RV-Modell A & 3 & 6 & 12 & 8 & 6 & 4 & 1 \\
\hline RV-Modell B & 1 & 3 & 2 & 7 & 5 & 10 & 12 \\
\hline RV-Modell C & 0 & 1 & 7 & 7 & 14 & 3 & 8 \\
\hline RV-Modell D & 1 & 10 & 7 & 10 & 5 & 5 & 2 \\
\hline RV-Modell E & 1 & 9 & 16 & 8 & 4 & 0 & 2 \\
\hline$\sum$ aller Modelle & $6=3 \%$ & $29=14,5 \%$ & $44=22 \%$ & $40=20 \%$ & $34=17 \%$ & $22=11 \%$ & $25=12,5 \%$ \\
\hline
\end{tabular}

\begin{tabular}{|c|c|c|c|c|c|c|c|}
\hline Zeit (Min.) & $\mathbf{0}$ & $\mathbf{1}$ & $\mathbf{2}$ & $\mathbf{3}$ & $\mathbf{4}$ & $\mathbf{2 5}$ & k.A. \\
\hline RV-Modell A & 0 & 12 & $\mathbf{1 5}$ & $\mathbf{8}$ & $\mathbf{2}$ & 2 & 2 \\
\hline RV-Modell B & 0 & 10 & $\mathbf{1 6}$ & $\mathbf{1 2}$ & $\mathbf{1}$ & 0 & 0 \\
\hline RV-Modell C & 0 & 0 & $\mathbf{7}$ & $\mathbf{1 4}$ & $\mathbf{5}$ & 10 & 3 \\
\hline RV-Modell D & 0 & 1 & $\mathbf{1 8}$ & $\mathbf{1 5}$ & $\mathbf{1}$ & 5 & 1 \\
\hline RV-Modell E & 0 & 4 & $\mathbf{1 3}$ & $\mathbf{1 0}$ & $\mathbf{5}$ & 5 & 3 \\
\hline$\sum$ aller Modelle & $0=0 \%$ & $\mathbf{2 7 = 1 3 , 5 \%}$ & $\mathbf{6 9 = 3 4 , 5 \%}$ & $\mathbf{5 9 = 2 9 , 5} \%$ & $\mathbf{1 4 = 7} \%$ & $\mathbf{2 2 = 1 1} \%$ & $9=4,5 \%$ \\
\hline
\end{tabular}

Tabelle 5:

Zeitintervalle, in denen der RV-Schlussmechanismus einsetzte. Ergebnisse bei einer Förderrate von 0,1ml/h (oben) bzw. $1 \mathrm{ml} / \mathrm{h}$ (unten). K.A. Angabe war nicht möglich (z.B. Undichtes RV). Fett hinterlegte Angaben lagen im

Referenzbereich.

Nach RV-Schluss zeigten sich proportional zum verschobenen Volumen linear ansteigende Druckwerte. Bei 4 von 5 RV-Modellen zeigte sich, dass der Schluss der RVs verspätet bzw. gar nicht einsetzte. RV-Modell B zeigte insgesamt bei einer Förderrate von $0,1 \mathrm{ml} / \mathrm{h}$ am häufigsten verspätete lineare Verläufe.

\subsection{Ergebnisse der mikrobiologischen Testung der RVs}

\subsubsection{Testung der Verhinderung bakterieller Kontamination durch RVs bei Infusion gegen die RV-Durchflussrichtung}

Bei pumpengetriebener Infusion gegen die RV-Durchflussrichtung und geschlossenen RVs konnten $S$. aureus, $S$. epidermidis sowie $P$. mirabilis, die RVs und die sich anschließende Druckleitung kontaminieren. 
S. epidermidis:

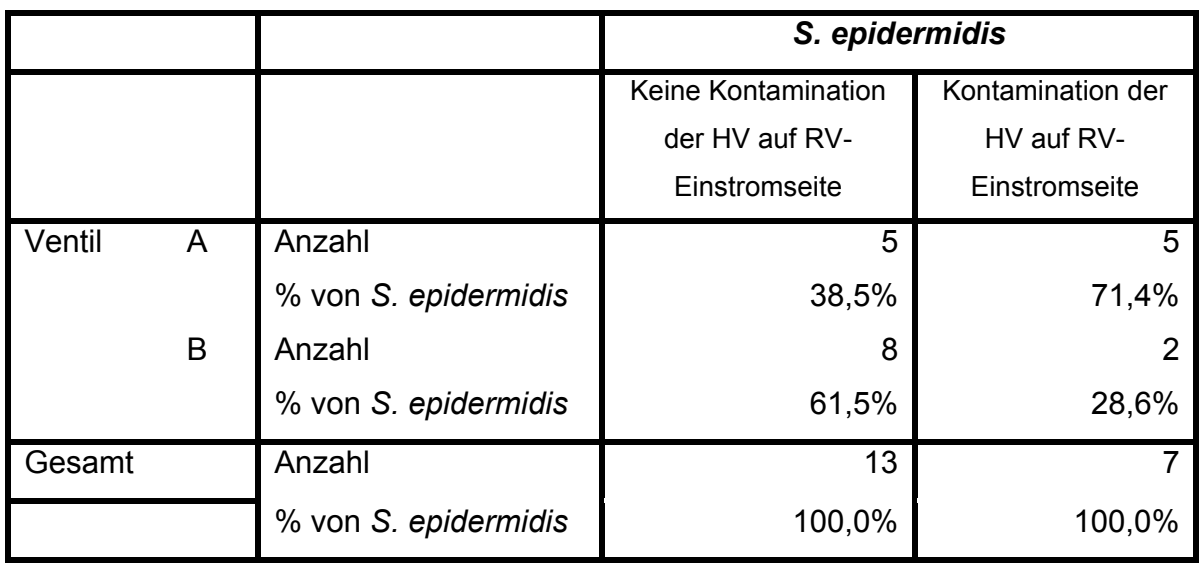

Tabelle 6: $\quad$ Häufigkeit einer bakteriellen Kontamination der Druckleitung (HV) durch Passage von S. epidermidis durch ein RV gegen die RV-Durchflussrichtung. $N=10$ Testungen pro RV-Modell.

Bei Ventilmodell A war bei 4 von 5 Testungen, bei denen eine Kontamination über das RV nachweisbar war, die Druckleitung über eine Länge von 2,3 cm kontaminiert. Bei einer RV-Testung waren die Druckleitung über $6,3 \mathrm{~cm}$ mit $S$. epidermidis kontaminiert. Insgesamt besiedelte $S$. epidermidis längere Abschnitte der Druckleitungen als $S$. aureus.

\section{S. aureus:}

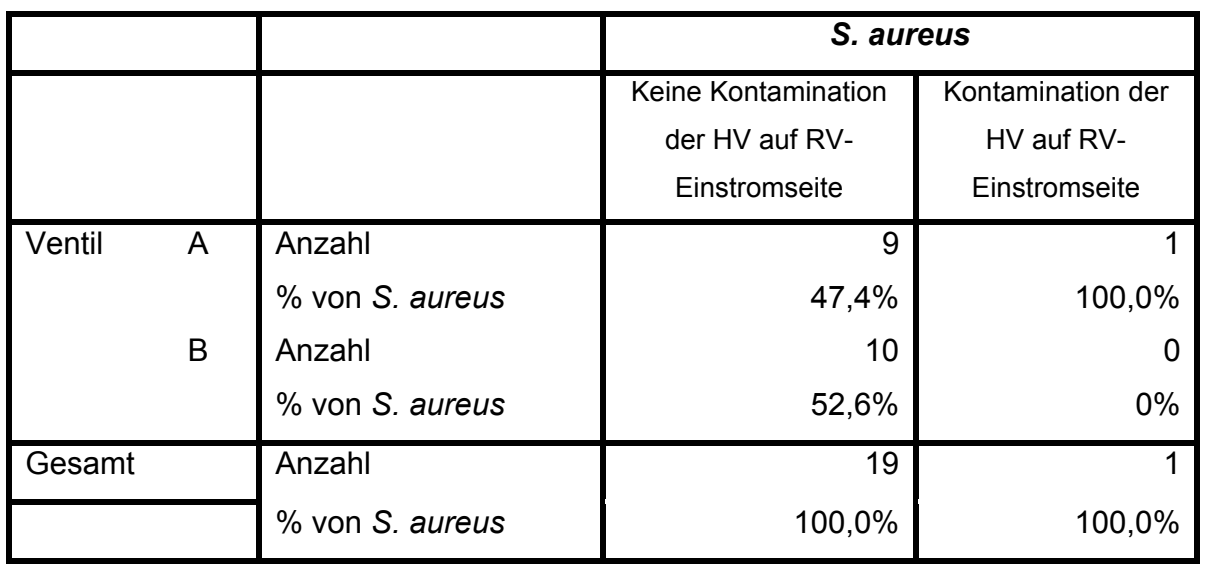

Tabelle 7: $\quad$ Häufigkeit einer bakteriellen Kontamination der Druckleitung (HV) durch Passage von S. aureus durch ein RV gegen die RV-Durchflussrichtung. $N=10$ Testungen pro RV-Modell. 
Bei Ventilmodell A war eine Kontamination im ersten Abschnitt der Druckleitung nachweisbar, entsprechend einer Länge der Druckleitung von 2,2 cm. Insgesamt war $S$. aureus der Keim, der am wenigsten weit die Druckleitung kontaminierte.

\section{P. mirabilis:}

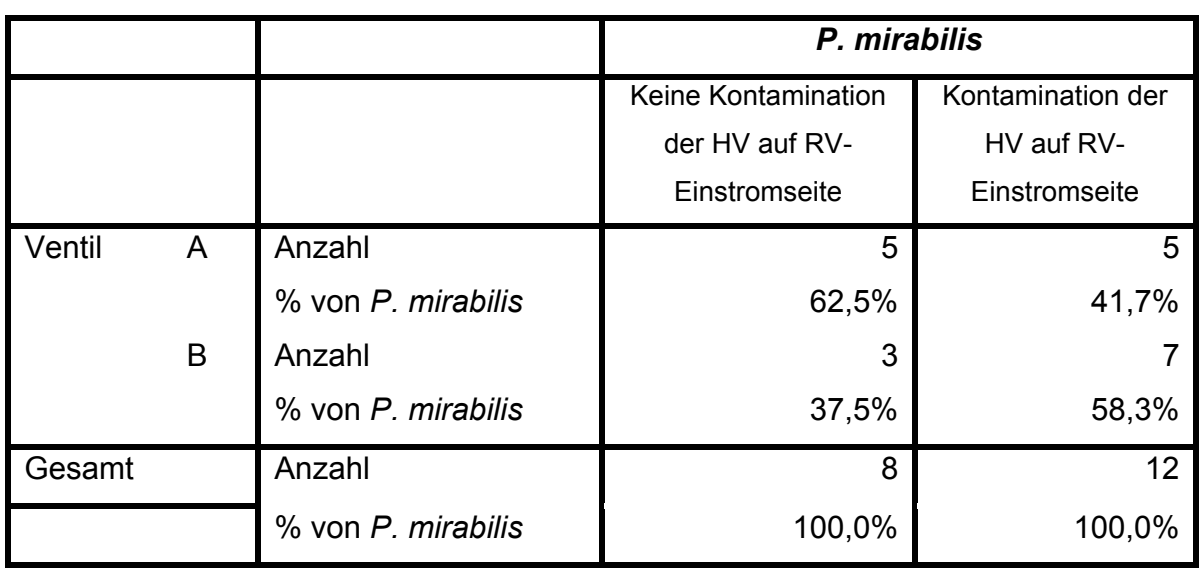

Tabelle 8: $\quad$ Häufigkeit einer bakteriellen Kontamination der Druckleitung (HV) durch Passage von P. mirabilis durch ein RV gegen die RV-Durchflussrichtung. $N=10$ Testungen pro RV-Modell.

Bei RV-Modell B war eine bakterielle Kontamination über das RV gegen die RVDurchflussrichtung über eine Länge von $2,7 \mathrm{~cm}$ in der Druckleitung nachweisbar. Bei RV-Modell A zeigte sich bei 2 Messungen eine Kontamination über eine Länge von 2,35 cm. Bei drei RV-Testungen über eine Länge von 5,5 $\mathrm{cm}$. $P$. mirabilis konnte verglichen mit $S$. epidermidis häufiger längere Abschnitte der Druckleitung gegen die RV-Durchflussrichtung kontaminieren.

Zusammenfassend konnte eine Kontamination der Druckleitungen durch RVModell B insgesamt 21mal verhindert werden. Neunmal konnten Bakterien das RV gegen die RV-Durchflussrichtung passieren. 19 RVs des Modells A verhinderten die bakterielle Kontamination. 11mal konnten Bakterien das Druckleitungssystem über das RV kontaminieren.

Im Folgenden sollte getestet werden, ob die Kontaminationshäufigkeit der einzelnen Bakterien über die RVs abhängig von dem getesteten RV-Modell war. 
Zur Signifikanztestung bei kleiner Fallzahl ( $n=10$ Testungen je Bakterienstamm je RV-Modell) wurde der nicht-parametrische exakte Test nach Fischer durchgeführt. Der exakte Test nach Fisher ergab bei $S$. epidermidis einen 2seitigen $p$-Wert von 0,350 . Bei $S$. aureus $p=1$ und bei Testung von $P$. mirabilis einen 2-seitigen $\mathrm{p}$-Wert von 0,65 .

\subsubsection{Testung bakterieller Kontamination retrograd über das RV gegen Flüssigkeitsstrom in RV-Durchflussrichtung}

Unser Experiment zeigte, dass Bakterien bei Flüssigkeitsstrom in RVDurchflussrichtung retrograd gegen den Flüssigkeitsstrom wandern und die Druckleitung nach Passage des RVs kontaminieren konnten. Bei keinem mit $\mathrm{NaCl}$ 0.9\%- und Propofol-Lösung durchgeführtem Versuch war die mit Infusionslösung gefüllte Perfusorspritze zu Versuchsbeginn, nach 24, 48 und 72 Stunden bakteriell kontaminiert.

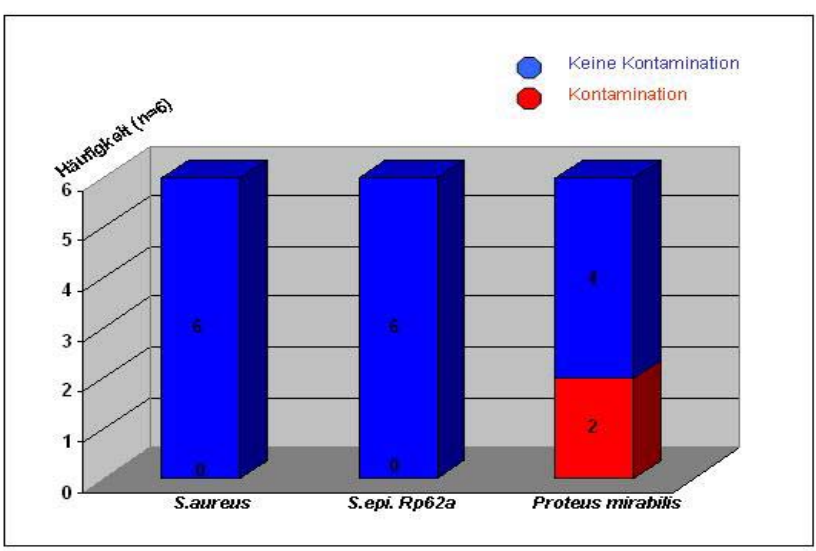




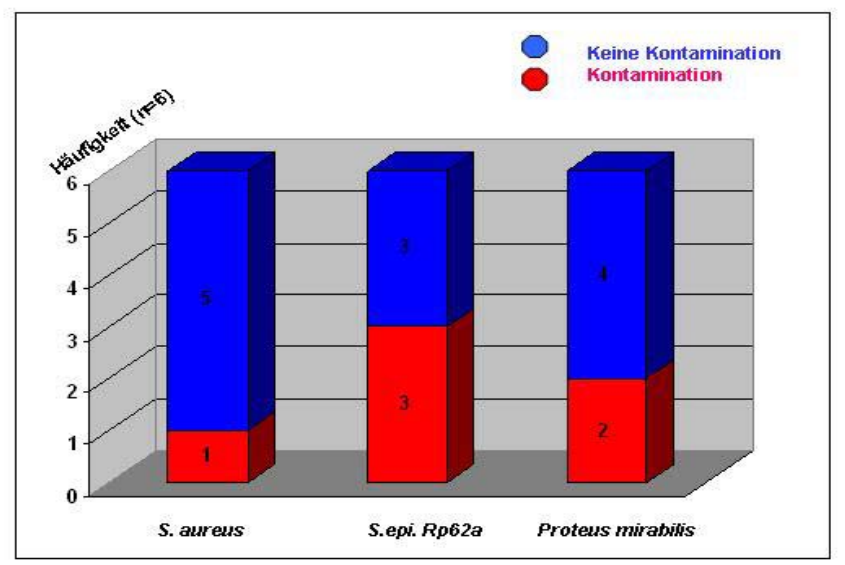

Abbildung 20: Anzahl an Kontaminationen (rot) versus keine Kontaminationen (blau) der Druckleitungen durch Bakterien, die das RV retrograd gegen einen Flüssigkeitsstrom in RV-Durchflussrichtung passieren konnten. $N=6$ Testungen pro Bakterienstamm. Verwendete Infusionslösungen im oberen Balkendiagramm NaCl $0.9 \%$, im unteren Balkendiagramm Propofol.

Bei kleiner Fallzahl getesteter RVs ( $n=6$ Testungen pro Bakterienstamm und pro Infusionslösung) wurde das Signifikanzniveau zum Nachweis einer unterschiedlichen Kontamination zwischen $\mathrm{NaCl} 0.9 \%$ und Propofol nicht erreicht (nicht-parametrische exakte Test nach Fisher; $S$. aureus $p=1 ; S$. epidermidis $p=0.1818$; $P$. mirabilis $p=1$ ). 


\section{Diskussion}

\subsection{Diskussion der physikalischen Testung der RVs}

\subsubsection{Diskussion der Methode}

\subsubsection{Prüfung der Hypothese und Literaturrecherche}

RVs zeigen in unserem Experiment bei einer Förderrate von 0,1 $\mathrm{ml} / \mathrm{h}$ gegen die RV-Durchflussrichtung eine Fehlerrate von 14\%. Bei einer Förderrate von 1 $\mathrm{ml} / \mathrm{h}$ betrug die Rate an RVs, die einen Rückfluss nicht verhinderten $9.5 \%$.

In der aktuellen Literatur finden sich zu RVs und deren Fehlerrate bei Anwendung im Rahmen von Infusionen keine Daten. Bei Recherche in der Datenbank "PubMed" finden sich unter den Suchbegriffen „one-way valves [ti]" 5 , „one-way check valves [ti]“ 7 , „check-valves [ti]“ 5 und „check-valves“ 32 Artikel, die sich fast ausschließlich mit Beatmungsventilen oder mit der Verwendung von Ventilen bei der Weiterverwendung von Kontrastmittelresten beschäftigen. Lediglich ein Artikel beschreibt einen Fallbericht von einem komplett versagenden RV im Rahmen einer Anästhesie mit Propofol (Crosby 1991). Crosby beobachtete, dass während der Narkose eines 60jährigen Mannes immer dann, wenn der Cuff der Blutdruckmanschette aufgepumpt wurde, ein Rückfluss von Propofol durch ein RV zu erkennen gewesen sei. Das RV schien intakt. Es waren keine Luftblasen sichtbar die das RV blockierten. Man erhöhte die Infusionsrate, wodurch der retrograde Fluss während der Blutdruckmessung suffizient unterbunden werden konnte.

\subsubsection{Klinische Relevanz des Experiments}

Unsere Hypothese, dass RVs eine bakterielle Kontamination von Druckleitungen nicht verhindern können, wurde mit unseren mikrobiologischen Testungen bewiesen.

Im ersten Versuchsteil wurde durch pumpengetriebene Infusion ein Druck gegen die RV-Durchflussrichtung aufgebaut. Im klinischen Alltag kann der 
Druck durch gleichzeitiges Aufpumpen einer Manschette für eine nicht-invasive Blutdruckmessung (NIBP) nach Riva-Rocci an einem für eine total intravenöse Anästhesie verwandten Arm oder durch einen stark angewinkelten Arm, der die Druckleitungen intermittierend abknickt, induziert werden. Die Anwender von RVs in Infusionssystemen gehen davon aus, durch das RV einen Rückfluss von Flüssigkeit und Bakterien vom Patienten zur Infusionslösung effektiv verhindern zu können.

Wenn für eine kurze Narkose nur geringe Medikamentenmengen benötigt werden, ist in der Praxis denkbar, dass Propofol mehreren Patienten aus derselben Ampulle infundiert wird. Dabei wird ein RV an das Infusionssystem konnektiert, unter der Vorstellung durch das RV einen retrograden Flüssigkeitsund Bakterienfluss und damit eine Kontamination der Restlösung zu verhindern und anschließend eine Weiterverwendung der Medikamentenreste bei weiteren Patienten zu rechtfertigen. Die Anwendung von 50ml-Propofo/perfusoren gehört bei Intensivpatienten zur Routine. Je nach Förderrate der Perfusoren werden diese Substanzen in Abhängigkeit vom Bedarf des Patienten entgegen der Empfehlung der Fachinformation evtl. auch länger als 12 Stunden verwendet.

Unsere Daten belegen, dass RVs weder einen Rückfluss von Flüssigkeiten gegen die RV-Durchflussrichtung noch eine bakterielle Kontamination verhindern können und verdeutlichen die Gefahr von o.g. Vorgehen im klinischen Alltag.

§ 23 Abs. 3 des am 01. Januar 2001 mit dem Gesetz zur Neuordnung seuchenrechtlicher Vorschriften in Kraft getretenen Infektionsschutzgesetzes sieht vor, dass das Robert-Koch-Institut (RKI) über eine Kommission für Krankenhaushygiene und Infektionsprävention verfügt. Diese erarbeitet Empfehlungen, die auf einem breiten, fachlichen Konsens basieren. Von Experten formulierte Kommissionsempfehlungen werden erst nach Anhörung durch Länder, Verbände und Körperschaften durch das RKI publiziert. Die Empfehlungen stellen kein verbindliches Recht dar, gelten gemäß aktuellem Stand des Wissens aber als "State of the Art" (Robert-Koch-Institut, 2006). In den Richtlinien für Krankenhaushygiene des RKIs findet u.a. die Verwendung von Infusionssystemen Berücksichtigung. Die im vorliegenden Experiment 
untersuchten, häufig verwendeten RVs werden nicht erwähnt (Robert-KochInstitut, Bundesgesundheitsblatt, 2002). Daher ist die korrekte Verwendung dieser Infusionssystemkomponenten häufig unklar und nicht explizit geregelt. Da eine Gefahr bakterieller Kontamination von Infusionslösungen trotz RVs besteht, könnte eine Anpassung der RKI-Empfehlungen erwogen werden.

\subsubsection{Versuchsaufbau im Vergleich zur DIN-Norm 58362}

Der Versuchsaufbau unseres Experiments war eine modifizierte Variante des gemäß DIN-Norm 58362 entwickelten Versuchsaufbaus zur physikalischen Überprüfung des Sperrverhaltens von RVs. Die vom Normenausschuss Medizin (NAMed) im Deutschen Institut für Normung e.V. (DIN) erstellte DIN-Norm 58362 befasst sich mit „Infusionsgeräten und deren Zubehör“. Der 7. Teil dieses Normenentwurfs befasst sich explizit mit sterilisierten, zur einmaligen Verwendung bestimmten RVs, die zusammen mit Infusionsgeräten für Schwerkraftinfusion und/oder Druckinfusionsapparate verwendet werden (DINNorm 58362-7, 2000).

Um das Schließverhalten der RVs bei pumpengetriebener Infusion gegen die RV-Durchflussrichtung zu testen, wird nach DIN-Norm Abschnitt A.1.1.6. gegen die RV-Durchflussrichtung ein kontinuierlicher Flüssigkeitsüberdruck von 200 $\mathrm{kPa}(=1.500,12 \mathrm{mmHg})$ aufgebaut. Die Prüfung wird bei $(23+/-1)^{\circ} \mathrm{C}$ sowie $(40+/-$ $1)^{\circ} \mathrm{C}$ für 15 Minuten durchgeführt. Dabei soll festgestellt werden, ob das RV suffizient schließt (DIN-Norm, 2000).

Da die RV-Anwendung meist bei Raumtemperatur stattfindet, wurde unser Experiment analog zur Anwendung gemäß DIN-Norm 58362 bei einer Temperatur von $(23+/-1)^{\circ} \mathrm{C}$ durchgeführt. Die RVs wurden in unserem Experiment jedoch nicht einem kontinuierlich hohen Druck von $200 \mathrm{kPa}$ $(=1.500,12 \mathrm{mmHg})$ ausgesetzt, da dieses Druckniveau im klinischen Gebrauch nicht relevant ist. Das RV-Schließ- bzw. Ansprechverhalten wurde bei dynamisch zunehmendem Druck durch Volumenverschiebung über eine pumpengetriebene Infusion mit einer Förderrate von 0,1 bzw. $1 \mathrm{ml} / \mathrm{h}$ getestet, da dieses die kritische, klinische Situation besser reflektiert und in den 
Herstellerinformationen der RVs der minimale Schliessfluß mit $\leq 0,1 \mathrm{ml} / \mathrm{h}$ gegen die RV-Durchflussrichtung angegeben wird. Den RV-Schlussmechanismus bei geringem Gegendruck zu testen, ist eine genauere Prüfung des RVSchließverhaltens, da im Vergleich der beiden Gruppen mit unterschiedlichen Förderraten der Schliessmachanismus schneller in der Gruppe mit einer höheren Förderrate gegen die Durchflussrichtung einsetzte.

Der von uns entwickelte Versuchsaufbau war ein geschlossenes System. Mit einem Druckmesser für invasive Blutdruckmessung wurden Druckanstiege/abfälle bei kontinuierlicher pumpengetriebener Infusion gemessen. Flüssigkeit fließt in Richtung des geringsten Widerstandes. Bei einer Flüssigkeitsverschiebung über ein undichtes RV wird durch Volumenverlust auf der einen Seite ein Druckabfall induziert. Die visuelle Beurteilung eines Flüssigkeitsspiegels durch „abfallender Tropfen“- wie in der Prüfung nach DINNorm, ist weniger sensitiv als die Messung von Druckanstiegen/-abfällen. Daher halten wir unsere Testung des RV-Schließverhaltens für exakter als die Testung nach DIN-Norm.

\subsubsection{Einfluss von $\mathrm{NaCl} 0.9 \%$-Lösung auf die Experimente}

Auf Grund kristalloider Eigenschaften der $\mathrm{NaCl}$ 0.9\%-Lösung ist denkbar, dass Salzgitterstrukturen zu einer Schädigung der RV-Membran/Feder oder zu einer anderweitigen Blockade des RVs führen könnten. Um dieses auszuschließen wurde für jeden Versuch ein neues RV in den Versuchsaufbau eingebaut. Die undichten Ventile konnten im mikrobiologischen Teil auf Grund von vorheriger Kontamination nicht erneut verwendet werden.

\subsubsection{Diskussion der Kurvenverläufe und Steigungsbeginne}

Ein dichtes RV verhindert eine Volumenverschiebung gegen die RVDurchflussrichtung. Der Druckmesser zeigte bei dichtem RV, einen kontinuierlichen, proportional zur Förderrate der pumpengetriebenen Infusion 
ansteigenden Druck. Kurven mit einem „langsamen Anstieg und verspäteten linearen Verlauf" waren bedingt durch die Mechanik der Infusionspumpe. Die Perfusoren förderten erst nach einer Vorlaufzeit bestimmte Volumina mit einer stabilen Förderrate, da an der Stelle des Übergangs vom Kolben des Perfusors zum Kolbenschieber der Kolben zuerst einrasten muss, bevor effektiv Volumina gefördert werden können.

Für spezifische Infusionspumpen in Kombination mit Perfusorspritzen existieren Trompetenkurven (Anhang Grafik I), die das Anlaufverhalten des Pumpenantriebes nach Start einer Infusion zeigen. Trompetenkurven demonstrieren die minimalen und maximalen Abweichungen von der Förderratengenauigkeit bei definierter Kombination Spritze/Spritzenpumpe. Insbesondere bei kleinen Förderraten treten erhebliche, weit über den angegebenen Genauigkeiten liegende Fehler bei der Förderrate auf. Insbesondere bei kurzzeitigem oder intermittierendem Betrieb der Infusionspumpen können diese Abweichungen bedeutsam sein (Hintzenstern U.v. 2004). Je höher die Förderrate desto geringer der relative Fehler. Die Förderrate stabilisiert sich, je länger die Pumpe in Betrieb ist, so dass mit zunehmender Versuchszeit der Anteil der prozentualen Fehlförderung abnimmt. Die Mittelwerte unserer Druckkurven bei RV-Testung zeigten, dass die Kurven bei einer Förderrate von 0,1 ml/h durchschnittlich nach 8-24 Minuten anstiegen (Förderrate $1 \mathrm{ml} / \mathrm{h}$ nach 1-2 Minuten).

\subsection{Diskussion der mikrobiologischen Testung der RVs}

Im vorliegenden Experiment wurde gezeigt, dass Bakterien Druckleitungen auch bei Verwendung von RVs kontaminieren konnten. Es wurde gezeigt, dass Bakterien RVs passieren konnten, wenn ein Flüssigkeitsstrom gegen die RVDurchflussrichtung aufgebaut wurde und die RVs in geschlossenem Zustand sein sollten. Auch bei einem Flüssigkeitsstrom in RV-Durchflussrichtung, konnten Bakterien retrograd gegen den Flüssigkeitsstrom das RV passieren und die sich anschließende Druckleitung kontaminieren. 
RVs stellen keinen infektiologischen Schutz von Infusionslösungen dar. Durch Einsatz von RVs kann daher eine Weiterverwendung von Infusionslösungsresten bei unterschiedlichen Patienten nicht gerechtfertigt werden.

\subsubsection{Passage von S. epidermidis durch RVs}

S. epidermidis konnte beide getesteten RV-Modelle passieren. Als ursächlicher Faktor für die Passage von $S$. epidermidis ist die Fähigkeit dieses Bakterienstammes zur Biofilmbildung denkbar. Nachdem erkannt worden war, dass die zur kommensalen Flora der Haut und Schleimhaut des Menschen gehörigen koagulasenegativen Staphylokokken, die bei immunkompetenten Menschen meist apathogen sind (Kloos 1997; Noble 1997) auch pathogene Eigenschaften haben, begann die Suche nach verantwortlichen Faktoren. Ein möglicher Zusammenhang zwischen einer Schleimproduktion sowie einer Besiedlung von Fremdkörpern, die gemeinsam zu einer Infektion führen könnten, wurde erstmals von Christensen et al. (1982) diskutiert. Heute spricht man zunehmend von „Biofilm“, da der zuvor häufig verwandte Begriff „Schleim“ oft unterschiedlich definiert worden ist. Es folgten Studien, die den Zusammenhang zwischen Biofilmbildung und der klinischen Relevanz von Erregern, die zur Biofilmbildung fähig waren, aufzeigten (Ponce de Leon et al. 1986; Righter 1987; Skoutelis et al. 1990). Auch konnte gezeigt werden, dass ein Zusammenhang zwischen der Bildung von Biofilmen und dem Versagen therapeutischer Maßnahmen bestehe (Archer 1994; Beaman et al. 1987; DiazMitoma et al. 1987; Kristinsson et al. 1986; Raad et al. 1998). In Biofilmen angeordnete Bakterien weisen erhöhte Resistenzen gegen extern einwirkende Kräfte auf. Wir konnten zeigen, dass an den Grenzflächen der Druckleitungen adhärierende Bakterien den Scherkräften, die durch Flüssigkeitsstrom in RVDurchflussrichtung entstehenden waren, standhalten konnten und so auch gegen einen Flüssigkeitsstrom wuchsen und das Druckleitungssystem kontaminieren konnten. 


\subsubsection{Passage von S. aureus durch RVs}

Insgesamt konnte $S$. epidermidis häufiger als $S$. aureus die RVs passieren. Beide Bakterien gehören zur Spezies der Staphylokokken und sind in vielen Eigenschaften identisch. Die beiden Stämme unterscheiden sich jedoch in der Fähigkeit zur Biofilmbildung. Dieses Ergebnis legen nahe, dass die Fähigkeit von S. epidermidis zur Biofilmbildung entlang der Druckleitungen für die Kontamination der relevante Mechanismus war und nicht die passive Ausbreitung in der Infusionsflüssigkeit.

Da $1 / 3$ aller nosokomialen Infektionen auf Infektionen mit $S$. aureus zurückzuführen sind, wurde in unserem Experiment das Verhalten dieses Bakteriums betrachtet. Zudem gewinnt dieser Keim aufgrund kontinuierlich ansteigender Fallzahlen von Methicillin-Resistenten-Staphylococcus-aureus (MRSA) zunehmend an Bedeutung. 1995 betrug die MRSA - Prävalenz in Zentraleuropa (inklusive Deutschland) 8,7\%. Verglichen mit 1990 impliziert dies nach Witte et al. (1997) einen Anstieg um $+7 \%$ (Witte et al. 1997). Mittels Krankenhaus-Infektions-Surveillance-System (KISS) werden Daten aus über 200 Intensivstationen in Deutschland erfasst. Die Häufigkeit von MRSA als Anteil aller $S$. aureus-Isolaten innerhalb dieses Erfassungssystems stieg im Mittel von 8\% im Jahr 1997 auf 30\% im Jahr 2003. Der Anteil von MRSA lag dabei bei der primären S. aureus-Sepsis bei $37,8 \%$ und beispielsweise bei der nosokomialen Pneumonie bei 21,5\% (Robert-Koch-Institut, Epidemiologisches Bulletin 04.Februar 2005).

\subsubsection{Passage von $P$. mirabilis durch RVs}

P. mirabilis scheint insgesamt am häufigsten eine Kontamination der Druckleitungen zu induzieren, auch wenn das statistische Signifikanzniveau wahrscheinlich auf Grund des kleinen Stichprobenumfangs nicht erreicht wurde. $P$. mirabilis ist sehr beweglich und ein Keim mit sehr kurzer Generationszeit. Das durch peritriche Begeißelung bedingte Schwärmphänomen ermöglicht $P$. mirabilis eine gute und schnelle Fortbewegung. Die Migration eines schnell 
bewegenden Keims führte in unseren Experimenten innerhalb kürzester Zeit zu einer Kontamination der sich anschließenden Druckleitungen sowie der Infusionslösungen. Hersteller B erwähnte auf explizite schriftliche Anfrage, dass bei Anwendung von RVs die Kontamination mit "nicht sehr beweglichen Keimen" ein Aufsteigen potentieller Erreger ermögliche und eine Gefahr darstelle. Diese Aussage konnte in unserem Experiment wiederlegt werden. Der besonders bewegliche Keim $P$. mirabilis konnte selbst in $\mathrm{NaCl} 0.9 \%$, welches ein schlechtes Nährmedium für Bakterien darstellt, das RV passieren und die Druckleitung kontaminieren. Da in der Information vom Hersteller B keine Literaturangaben gemacht wurden, konnte nicht nachvollzogen werden, worauf diese Aussage des Herstellers basierte.

\subsection{Limitationen unseres Experiments}

Zunächst besteht eine Limitation unseres Experimentes sicherlich in der geringen Anzahl getesteter RVs. Ggf. würden sich bei Testung größerer Fallzahlen mit ausreichender statistischer Power, signifikante Unterschiede zwischen der bakteriellen Kontamination über RVs von unterschiedlichen Bakterien zeigen. Auch Qualitätsunterschiede zwischen den einzelnen RVModellen hinsichtlich der effektiven Verhinderung von Rückfluss von Infusionslösungen wären bei größerer Fallzahl ggf. signifikant. Ziel unseres Experiments war jedoch nachzuweisen, dass RVs weitgehend unabhängig vom Hersteller, keinen zuverlässigen Schutz gegen bakterielle Migration bieten, und nicht um die exakte Quantifizierung der möglichen Inzidenz oder dem Belegen von Produktunterschieden.

Um eine Biofilmbildung von S. epidermidis nachzuweisen, wäre ein Tube-Test notwendig gewesen. Technisch konnte der Tube-Test in unserem Experiment nicht durchgeführt werden. Die Druckleitungen wurden in unserem Experiment für 48 Stunden in Bouillon bebrütet. In dieser Bebrütungszeit konnten sich die ggf. in der Probe enthaltenen Bakterien unter optimalen Temperaturbedingungen im Reagenzglas vermehren. Hierdurch hätte das Ergebnis eines gleichzeitig durchgeführten Tube-Tests falsch positiv werden 
können. Unsere Ergebnisse mit größerer Anzahl an Kontaminationen durch $S$. epidermidis als durch $S$. aureus legen jedoch nahe, dass die größere Zahl an Kontaminationen am ehesten durch Biofilmbildung des adhärenten Staphylokokkenstammes bedingt ist. Ob die Biofilmbildung zur Kontamination der Druckleitungen führte, konnte mit unserem Versuchsaufbau nicht endgültig bewiesen werden.

Um die Anzahl der Kontaminationsquellen im Experiment zu reduzieren, wurden im mikrobiologischen Versuchsteil keine Druckmessungen durchgeführt. Dieses wäre interessant gewesen, um nachzuweisen, ob Bakterien nur undichte RVs oder auch dichte RVs passieren konnten. Mit unserem Versuchsaufbau konnte letztlich nicht geklärt werden, warum Bakterien die RVs passieren konnten. Denkbar wäre, dass das RV nicht effektiv schließt, so dass Bakterien permanent oder temporär das RV passieren könnten und das Druckleitungssystem kontaminieren könnten. Der Schlussmechanismus des RVs könnte auch verspätet reagieren, da das bei Perfusorstart verschobene Volumen möglicherweise zu gering ist, um einen Druck zu induzieren, der groß genug ist, um den RV-Schlussmechanismus auszulösen. In diesem Fall könnten Bakterien ebenfalls kurzzeitig das RV passieren und das Druckleitungssystem nach Vermehrung kontaminieren. Eine weitere Hypothese ist, dass das RV physikalisch zwar dicht wäre, die Poren der Feder jedoch so groß sind, dass eine Passage der Bakterien nicht verhindert werden kann. Die Filter der RVs könnten so zusammengesetzt sein, dass bei Kontakt mit der bakteriell kontaminierten Flüssigkeit chemische Prozesse stattfinden, die die Membran verändern und für Bakterien durchlässig machen könnten. Um den zugrunde liegenden Mechanismus endgültig zu klären sind weitere Versuche notwendig.

Da schon bei bakterieller Kontamination erhebliche Mängel deutlich werden, ist es sicherlich interessant in Zukunft die Effizienz dieser RVs hinsichtlich des Migrationsverhaltens von Viren näher zu betrachten. Aufgrund ihrer geringeren Größe und aufgrund eingeschränkter medikamentöser antiviraler Therapie stellen Viren eine bedeutsame Gefahr für den Patienten dar. 


\subsection{Zusammenfassende Beurteilung der Rückschlagventile}

Das vorliegende Experiment zeigte, dass die Fehlerrate an RVs, die einen Rückfluss von Infusionslösungen nicht sicher verhindern können, hoch ist. RVs können eine Passage von Bakterien nicht zuverlässig verhindern.

Einige Hersteller geben einen minimalen Schließfluss gegen die RVDurchflussrichtung von $\leq 0,1 \mathrm{ml} / \mathrm{h}$ an, bei dem das $\mathrm{RV}$ schließen und einen Rückfluss verhindern soll. $0,1 \mathrm{ml} / \mathrm{h}$ Volumenverschiebung entsprechen gleichzeitig dem geringsten mit den Perfusoren zu fördernden Volumen. In dem vorliegenden Experiment wurde das Schließverhalten der RVs bei minimalen Förderrate von 0,1 ml/h getestet, da dies auf Intensivstationen die Förderrate an Perfusoren ist, die im Standby-Modus bei temporär nicht verwandten Medikamentenperfusoren einprogrammiert wird, um deren Verfügbarkeit in akuten Situationen zu gewährleisten. Wenn Bakterien in eine Perfusorspritze gelangt sind, können sie sich dort vermehren. Die anschließende Applikation kann eine Bakteriämie induzieren, die bis zum Tod des Patienten führen kann. Neben der Förderrate von $0,1 \mathrm{ml} / \mathrm{h}$ wurde eine Förderrate von $1 \mathrm{ml} / \mathrm{h}$ untersucht. Es konnte gezeigt werden, dass nicht nur bei dem von einigen Herstellern als minimalem Schließfluss angegebenen Flussraten RVs nicht zuverlässig schließen, sondern dass dieses auch bei einer 10fach höheren Förderrate der Fall war. Eine Förderrate von $1 \mathrm{ml} / \mathrm{h}$ wird im klinischen Alltag häufig zur Medikamentenapplikation verwandt. Dies belegt die klinische Relevanz und mögliche Übertragung unserer Testungen auf den klinischen Alltag. Der Einsatz von RVs im klinischen Alltag impliziert bei den Anwendern möglicherweise eine nicht vorhandene Sicherheit der Verhinderung von Rückfluss und bakterieller Kontamination. Dem Ergebnissteil unseres Experimentes ist zu entnehmen, dass bei einer Förderrate von $0,1 \mathrm{ml} / \mathrm{h}$ von einer Fehlerrate nicht sicher schließender RVs von mindestens 14\% (28 Rückschlagventile; $\mathrm{n}=200$ ) ausgegangen werden muss (Förderrate 1,0 ml/h 7,5\% (15 Rückschlagventile; $n=200)$ ). Betrachtet man die Kurvenverläufe im Vergleich zum Verlauf der Referenzgeraden, ist die Rate der RVs, die eine Volumenverschiebung gegen die RV-Durchflussrichtung nicht sicher verhindern 
können sogar noch höher. Eine Durchflussminderung der Schwerkraftinfusion um bis zu $75 \%$ bedingt durch den zusätzlichen Widerstandes des zwischengeschalteten RVs stellt einen weiteren Nachteil bei der Verwendung von RVs dar (Herstellerinformation).

Unser Experiment zeigt, dass RVs keine Bakterienfilter sind. Dieser Hinweis ist den RV-Produktinformationen nicht zu entnehmen. Erst auf explizite Anfrage bei Hersteller B wurde erwähnt, dass die für Schwerkraftinfusionen produzierten RVs Bakterien aus dem Flüssigkeitsstrom nicht eliminieren oder deren Passage durch das RV nicht verhindern können. Die Verwendung von RVs gehört mittlerweile zum häufig praktizierten Standard bei Narkosen mit dem intravenösen Anästhetikum Propofol oder auch bei der Langzeitsedierung v.a. multimorbider, immunsupprimierter Patienten auf Intensivstationen. Wie die Studie von Halkes und Snow aus dem Jahre 2003 zeigt, ist vielen Anwendern unklar, welche Komponenten des Infusionssystems nach Anwendung bei einem Patienten verworfen werden müssen, und welche Komponenten zur Kostenreduktion bei anderen Patienten weiter verwendet werden dürfen. Halkes und Snow fanden mit Hilfe anonymer Fragebögen heraus, dass nur 13\% der Studienteilnehmer, bestehend aus Ärzten 10 verschiedener Krankenhäuser in Süd-West England sowie aus Mitgliedern der UK Gesellschaft für intravenöse Anästhesie (SIVA; Society for Intravenous Anaethesia UK), über die Existenz von Richtlinien informiert gewesen seien, welche die Wiederverwendung von TIVA-Narkose-Equipment betreffen. Die Studie zeigte eindeutig, dass diese Richtlinien in der Praxis selten adäquat beachtet werden. $46 \%$ der Studienpopulation tauschten unabhängig von der Restmenge an Propofol in der Perfusorspritze alle Komponenten des Infusionssystens von Patient zu Patient aus. Vermutlich waren sich diese Studienteilnehmer dem mit der Mehrfachverwendung von Infusionsmaterialen verbundenen erhöhten Infektionsrisiko bewusst. Die Mehrheit der Studienteilnehmer (69\%) verwendet einzelne Komponenten des Infusionssystems erneut bei anderen Patienten. Diese Gruppe scheint davon auszugehen, dass die Verwendung von RVs vor bakterieller Kontamination der sich anschließenden Medikamentenspritze schütze (Halkes u. Snow 2003). Unser Experiment zeigte allerdings, dass alle 
untersuchten Bakterienstämme dazu in der Lage waren RVs zu passieren und Druckleitungen zu kontaminieren.

Wie groß die Ausmaße der mit der Wiederverwendung von Infusionskomponenten verbundenen Risiken wie beispielsweise der Entwicklung einer Sepsis durch übertragene Bakterien sind, ist bisher nicht genau zu quantifizieren, da Daten in der Literatur fehlen.

In der aktuellen Literatur finden diese RVs kaum Berücksichtigung. Für die Verwendung dieser Komponenten eines Infusionssystems existieren keine definierten Handlungsanweisungen oder standardisierte Charakteristika.

Mit dieser Arbeit verdeutlichten wir die Ineffizienz von RVs. Zum einen konnte gezeigt werden, dass die Fehlerrate von RVs, die einen Rückfluss von Infusionslösungen nicht sicher verhindern konnte, hoch ist. Zudem konnte gezeigt werden, dass eine bakterielle Kontamination durch RVs nicht verhindert werden kann. 


\section{Literaturverzeichnis}

1. Archer, G. L. and M. W. Climo (1994) Antimicrobial susceptibility of coagulase-negative staphylococci. Antimicrob Agents Chemother 38(10): 2231-7

2. Arduino, M. J., L. A. Bland, et al. (1991) Microbial growth and endotoxin production in the intravenous anesthetic propofol. Infect Control Hosp Epidemiol 12(9): 535-9

3. AstraZeneca S.p.A. (2004), Gebrauchsanweisung Disoprivan $® 1 \%$ Wirkstoff. Propofol. AstraZeneca, London

4. Beaman, M., L. Solaro, et al. (1987) Peritonitis caused by slimeproducing coagulase negative staphylococci in continuous ambulatory peritoneal dialysis. Lancet 1(8523): 42

5. Christensen, G. D., W. A. Simpson, et al. (1982) Adherence of slimeproducing strains of Staphylococcus epidermidis to smooth surfaces. Infect Immun 37(1): 318-26

6. Crosby, E. (1991) Intravenous infusions and one-way valves. Can J Anaesth 38(6): 799-800

7. Diaz-Mitoma, F., G. K. Harding, et al. (1987) Clinical significance of a test for slime production in ventriculoperitoneal shunt infections caused by coagulase-negative staphylococci. J Infect Dis 156(4): 555-60

8. Halkes, M. J. and D. Snow (2003) Re-use of equipment between patients receiving total intravenous anaesthesia: a postal survey of current practice. Anaesthesia 58(6): 582-7 
9. Hein, H., R. Reinhardt, S. et al. (1987) Recapping needles in anesthesia safe? Anesthesiology 67: 161

10. Hintzenstern (2004) i.v. - Infusion, Transfusion und parenterale Ernährung. In: Hintzenstern i.v. - Infusion und parenterale Ernährung 3. Auflage (Hrsg) Urban \& Fischer

11.Kempen, P, Learned, D (1989) Anaesthesia practice - a vector for infection?. Anaesthesiology 71: A 948

12. Kloos W (1997) Taxonomy and systematics of staphylococci indigenous to humans. In: Crossley, K.B., Archer, G.L., et al. The staphylococci in human disease. New York: Churchill: Livingstone 113-137

13.Koepke, J, Reller, L, Master,H, Selner, J (1985) Contamination of intradermal skin test syringes. Ann Allergy 55: 776-778

14. Kramer A, Christiansen B, Exner M, Rotter M (2000) Händehygiene. Bundesgesundheitsbl Gesundheitsforsch Gesundheitssch 43: 644-648

15.Kristinsson, K. G., R. C. Spencer, et al. (1986) Clinical importance of production of slime by coagulase negative staphylococci in chronic ambulatory peritoneal dialysis. J Clin Pathol 39(1): 117-8

16.Kuehnert, M. J., R. M. Webb, et al. (1997) Staphylococcus aureus bloodstream infections among patients undergoing electroconvulsive therapy traced to breaks in infection control and possible extrinsic contamination by propofol. Anesth Analg 85(2): 420-5

17.Lessard, M, Trepaniere, C, Gourdeau, M, Denault, P (1988) A microbiological study of the contamination of the syringes used in anaesthesia practice. Can J Anaesth. 35: 567 - 569 
18. Noble, W. (1997) Staphylococcal carriage and skin and soft tissue infection. In: Crossley, K.B, Acher, G.L., ed. The staphylococci in human disease. New York: Churchill: Livingstone: 401-412

19.Ponce de Leon, S., S. H. Guenthner, et al. (1986) Microbiologic studies of coagulase-negative staphylococci isolated from patients with nosocomial bacteraemias. J Hosp Infect 7(2): 121-9

20.Raad, I. (1998) Intravascular-catheter-related infections. Lancet 351(9106): 893-8

21.Righter, J. (1987) Septicemia due to coagulase-negative Staphylococcus in a community hospital. Cmaj 137(2): 121-5

22. Robert-Koch-Institut (2005) Epidemiologisches Bulletin 04.02.2005 (Hrsg) Robert-Koch-Institut, Berlin

23.Skoutelis, A. T., R. L. Murphy, et al. (1990) Indwelling central venous catheter infections in patients with acquired immune deficiency syndrome. J Acquir Immune Defic Syndr 3(4): 335-42

24. Sosis, M. B. and B. Braverman (1993) Growth of Staphylococcus aureus in four intravenous anesthetics. Anesth Analg 77(4): 766-8

25.Sosis, M. B., B. Braverman, et al. (1995) Propofol, but not thiopental, supports the growth of Candida albicans. Anesth Analg 81(1): 132-4

26. Tessler, M., A. Dascal, et al. (1992) Growth curves of Staphylococcus aureus, Candida albicans, and Moraxella osloensis in propofol and other media. Can J Anaesth 39(5 Pt 1): 509-11 
27. Trepanier, C. A., M. R. Lessard, et al. (1990) Risk of cross-infection related to the multiple use of disposable syringes. Can J Anaesth 37(2): 156-9

28. Witte, W., M. Kresken, et al. (1997) Increasing incidence and widespread dissemination of methicillin-resistant Staphylococcus aureus (MRSA) in hospitals in central Europe, with special reference to German hospitals. Clin Microbiol Infect 3(4): 414-422 


\section{Lebenslauf}

Name:

Geburtsdatum:

Geburtsort:

Familienstand:

Staatsangehörigkeit:

Eltern:

Geschwister:

Schullaufbahn:

Studium:

Praktisches Jahr:
Daniela Kiski

04.11 .1980

Haltern

ledig

deutsch

Wolfgang Kiski

Luzia Kiski (geb. Homann)

André Kiski - geb. 04.11.1980

Christian Kiski - geb. 05.07.1983

Lisa Kiski - geb. 05.04.1993

1987 - 1991 Silverberg Grundschule Haltern

1991 - 2000 Städtisches Gymnasium Haltern

06/00 Abitur

10/00-12/06 Studium der Humanmedizin an der

Westfälischen Wilhelms-Universität Münster

12/06 Dritter Abschnitt der Ärztlichen Prüfung

1. Tertial: Innere Medizin; Klinik und Poliklinik für Kardiologie und Angiologie, Universitätsklinikum Münster, Münster

2. Tertial: Klinik und Poliklinik für Kinder- und Jugendmedizin, Universitätsklinikum Münster, Münster

3.Tertial: Michael E. DeBakey Department of Surgery; Baylor College of Medicine; Texas Medical Center; Houston, Texas (USA) 
Beruflicher Werdegang: 01/07-09/08 Wissenschaftliche Mitarbeiterin der Klinik und Poliklinik für Kardiologie und Angiologie; Universitätsklinikum Münster

Seit 10/08 Wissenschaftliche Mitarbeiterin der Klinik und Poliklinik für Pädiatrische Kardiologie; Universitätsklinikum Münster 


\section{Danksagung}

Für die Überlassung des Themas dieser Dissertation sowie die Unterstützung und Betreuung möchte ich mich ganz herzlich bei Herrn Univ.-Prof. Dr. med. Dr. h. c. H. Van Aken und Herrn Prof. Dr. med. F. Hinder bedanken.

Mein ganz besonderer Dank gilt speziell Herrn Priv.-Doz. Dr. med. B. Ellger und Herrn Priv.-Doz. Dr. med. A. Friedrich für Ihr außerordentliches Engagement bei der Betreuung und Korrektur dieser Arbeit.

Weiterhin möchte ich mich ganz herzlich bei Herrn Univ.-Prof. Dr. rer. nat. $\mathrm{H}$. Karch und dem Team aus dem Institut für Hygiene am Universitätsklinikum Münster sowie bei Herrn Dipl.-Ing. Thomas Morsell aus der Klinik und Poliklinik für Anästhesiologie für die vielen guten Ratschläge und Hilfestellungen bei der Durchführung der Versuche sowie für die Möglichkeit der Nutzung des Labors bedanken.

Insbesondere möchte ich zum Schluss auf diesem offiziellen Wege meinen Eltern und Geschwistern, denen diese Doktorarbeit gewidmet ist, danken. Sie haben mir diese Ausbildung ermöglicht, mir jederzeit und in jeder Hinsicht zur Seite gestanden, mich unterstützt und nicht zuletzt fortlaufend ermuntert, diese Doktorarbeit fertig zu stellen. 


\section{Anhang}

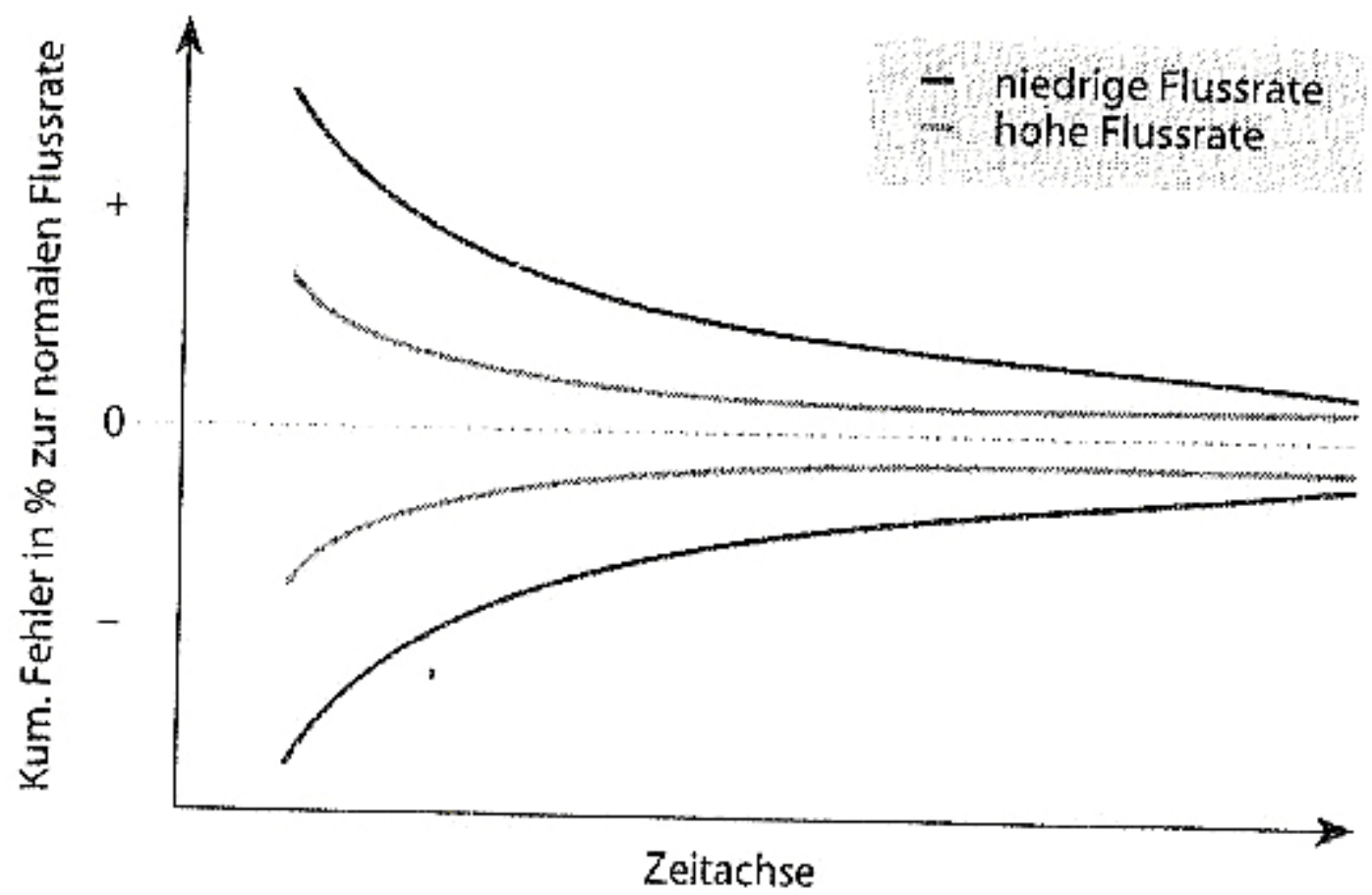

Grafik I: Trompetenkurven zeigen ein Anwachsen der prozentualen Fehlförderung von Infusionspumpen mit sinkender Infusionsrate der Infusionspumpe. (Hintzenstern U.v., 2004).
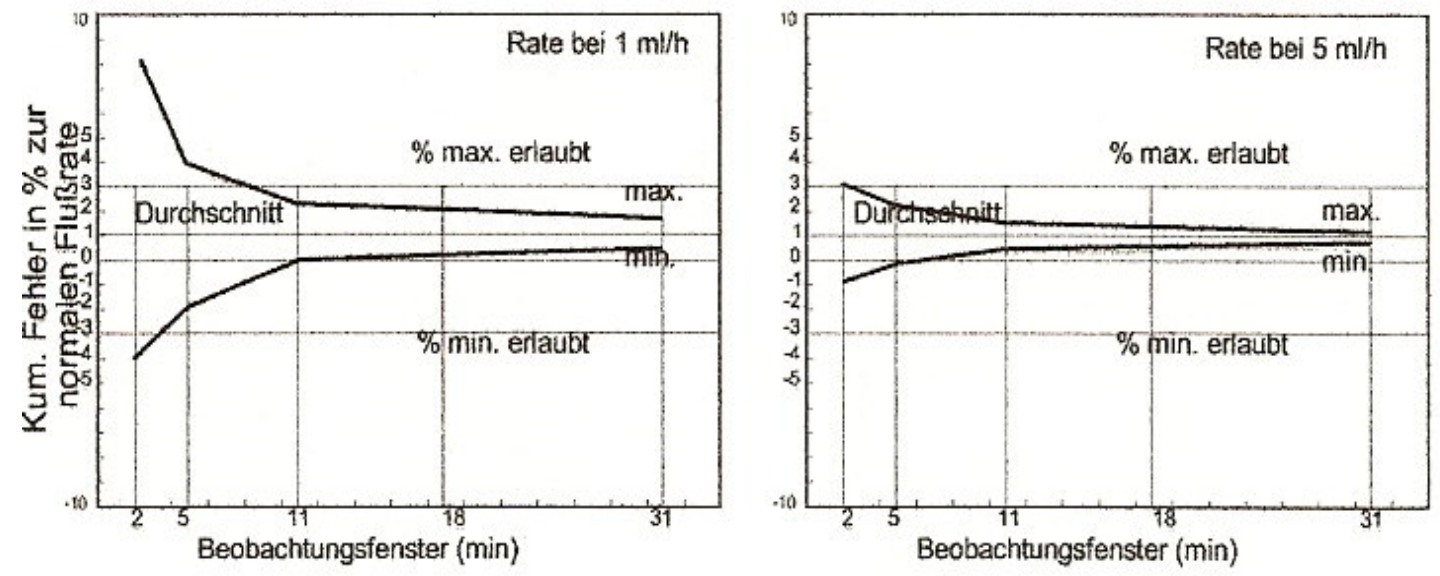

3enutzte Spritzen B-D Plastipak(B) 50 ml Luer Lok®. 

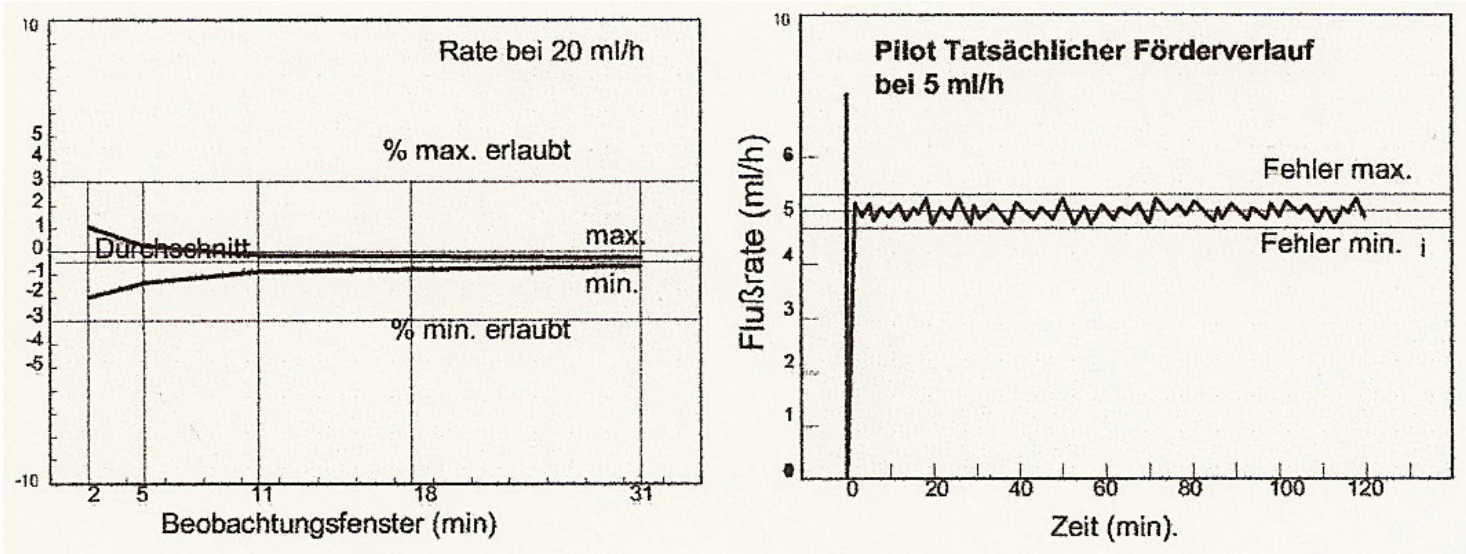

Grafik II: $\quad$ Trompetenkurve für BD-Spritzen Plastipak® 50mI Luer-Lock® 